\title{
DNA methylation signature of human fetal alcohol spectrum disorder
}

\author{
Elodie Portales-Casamar ${ }^{1 \dagger}$, Alexandre A. Lussier ${ }^{2 \dagger}$, Meaghan J. Jones ${ }^{2}$, Julia L. Maclsaac ${ }^{2}$, Rachel D. Edgar², \\ Sarah M. Mah ${ }^{2}$, Amina Barhdadi ${ }^{3}$, Sylvie Provost ${ }^{3}$, Louis-Philippe Lemieux-Perreault ${ }^{3}$, Max S. Cynader ${ }^{4}$, \\ Albert E. Chudley ${ }^{5,6}$, Marie-Pierre Dubé ${ }^{3,7}$, James N. Reynolds ${ }^{8}$, Paul Pavlidis ${ }^{1}$ and Michael S. Kobor $2,9^{*}$
}

\begin{abstract}
Background: Prenatal alcohol exposure is the leading preventable cause of behavioral and cognitive deficits, which may affect between 2 and $5 \%$ of children in North America. While the underlying mechanisms of alcohol's effects on development remain relatively unknown, emerging evidence implicates epigenetic mechanisms in mediating the range of symptoms observed in children with fetal alcohol spectrum disorder (FASD). Thus, we investigated the effects of prenatal alcohol exposure on genome-wide DNA methylation in the NeuroDevNet FASD cohort, the largest cohort of human FASD samples to date.

Methods: Genome-wide DNA methylation patterns of buccal epithelial cells (BECs) were analyzed using the Illumina HumanMethylation450 array in a Canadian cohort of 206 children (110 FASD and 96 controls). Genotyping was performed in parallel using the Infinium HumanOmni2.5-Quad v1.0 BeadChip.

Results: After correcting for the effects of genetic background, we found 658 significantly differentially methylated sites between FASD cases and controls, with 41 displaying differences in percent methylation change $>5 \%$. Furthermore, 101 differentially methylated regions containing two or more CpGs were also identified, overlapping with 95 different genes. The majority of differentially methylated genes were highly expressed at the level of mRNA in brain samples from the Allen Brain Atlas, and independent DNA methylation data from cortical brain samples showed high correlations with BEC DNA methylation patterns. Finally, overrepresentation analysis of genes with up-methylated CpGs revealed a significant enrichment for neurodevelopmental processes and diseases, such as anxiety, epilepsy, and autism spectrum disorders.
\end{abstract}

Conclusions: These findings suggested that prenatal alcohol exposure is associated with distinct DNA methylation patterns in children and adolescents, raising the possibility of an epigenetic biomarker of FASD.

\section{Background}

The prenatal environment has the potential to permanently imprint physiological and behavioral systems during development, leading to both short- and long-term health consequences. In particular, prenatal alcohol exposure (PAE) can alter the development, function, and

\footnotetext{
*Correspondence: msk@cmmt.ubc.ca

tElodie Portales-Casamar and Alexandre A. Lussier contributed equally to this work

${ }^{2}$ Department of Medical Genetics, Centre for Molecular Medicine and Therapeutics, Child and Family Research Institute, University of British Columbia, Vancouver, BC, Canada

Full list of author information is available at the end of the article
}

regulation of numerous neural and physiological systems, resulting in a variety of deficits falling under the umbrella of fetal alcohol spectrum disorder (FASD) [1]. Over the lifetime, the effects of PAE are manifested through cognitive and behavioral deficits, persistent alterations to stress responsivity and immune function, and increased vulnerability to mental health disorders and other comorbidities in individuals with FASD [1-4]. However, the degree to which alcohol exposure causes alterations during development varies, depending on factors such as timing and level of exposure, overall maternal health and nutrition, and genetic background [5]. As such, only a small proportion of affected children present with the 
phenotype of fetal alcohol syndrome (FAS), which is distinguished by growth deficits and facial dysmorphisms in addition to central nervous system dysfunction $[6,7]$. Nevertheless, the vast majority of children with FASD display physiological and neurobehavioral impairments lasting into adulthood, suggesting persistent programming effects of PAE across the spectrum of FASD [8].

While the etiology of the FASD currently remains unclear, epigenetics is emerging as an attractive candidate for the biological embedding of prenatal and early-life experiences in general and thus is a promising avenue for the study of FASD [9]. Epigenetics refers to modifications of DNA and its packaging that alter the accessibility of DNA to potentially regulate gene expression and cellular function without changes to the underlying genomic sequences [10]. The most studied epigenetic modification in human populations is DNA methylation, which refers to the covalent attachment of a methyl group to the $5^{\prime}$ position of cytosine, typically occurring in the context of cytosine-guanine dinucleotide (CpG) sites [11]. CpG sites are relatively rare in the human genome, yet do not occur at random; regions containing higher than expected levels of these dinucleotides have been termed "CpG islands" (CGIs) [12]. The 2-kb regions flanking CGIs are known as CGI "shores," while the areas located beyond shores are known as "shelves" [13-15]. Of note, these regions are typically more variable than CGIs themselves, as they have a greater range of DNA methylation across individuals [14]. DNA methylation is associated with the regulation of gene expression, although its effects on transcription are highly dependent on genomic context. For example, when located within gene promoters, DNA methylation generally represses gene expression, but this relationship is less well defined for CpGs located within gene bodies and intergenic regions [16]. Furthermore, DNA methylation is closely associated with several key developmental processes, including genomic imprinting, tissue specification and differentiation $[17,18]$. DNA methylation patterns are also population specific, as many $\mathrm{CpG}$ sites are associated with ethnicity [19-21]. There are a number of possible reasons for this association, including shared environments or associations of epigenetic marks with specific genetic variants [22-24].

Importantly, DNA methylation is malleable in response to environmental factors, and these changes may be inherited through cell divisions, potentially persisting throughout the lifetime [25-27]. For example, prenatal exposure to cigarette smoke is associated with longterm changes in DNA methylation of the AHRR gene, and maternal undernutrition during pregnancy leads to altered DNA methylation of IGF2 [28, 29]. Several studies have also characterized epigenetic changes following prenatal and postnatal ethanol exposure [30-36]. Early work in pregnant mice demonstrated that acute ethanol exposure during mid-gestation (gestational days 9-11) causes global genomic loss of DNA methylation in the fetus [37]. However, recent studies of embryonic cultures exposed to ethanol show that rather than a global demethylation of the genome by ethanol, some regions become more methylated and others less methylated [38]. Moreover, genome-wide studies in adult mice that were exposed to ethanol prenatally have also identified widespread changes in DNA methylation patterns in the entire brain, further suggesting an important role for epigenetics in the etiology of FASD [39]. Finally, a recent study characterized the DNA methylation profile in buccal epithelial cells (BECs) from a small cohort of human FASD samples, identifying alterations in the epigenome of children with FASD, particularly within the protocadherin gene clusters [40].

Collectively, these findings support epigenetic mechanisms as potential contributors to the deficits observed following PAE. However, no large-scale investigations of DNA methylation in individuals with FASD have been performed to date. In order to ascertain the effect of PAE on the human epigenome, the present study investigated the DNA methylation patterns of BECs from 110 children with FASD and 96 age- and sex-matched controls, to our knowledge representing the largest investigation on PAE effects on the human epigenome. Statistically significant alterations between FASD cases and controls were successfully identified following ethnic background correction, with a number of differentially methylated sites and regions located in genes previously associated with alcohol exposure $[38,40]$. Taken together, these results support a potential role for DNA methylation in the etiology of the neurobiological deficits observed in children with FASD and represent a potential epigenetic signature of FASD.

\section{Results}

The NeuroDevNet FASD epigenetics cohort

Participants in the NeuroDevNet Canadian FASD study cohort were recruited from six clinical sites across Canada (Vancouver, BC; Edmonton, AB; Cold Lake, AB; Winnipeg, MB; Ottawa, ON; and Kingston, ON) [39]. More specifically, 110 children with FASD or confirmed PAE and 96 typically developing controls were matched for sex and age, ranging from 5 to 18 years of age, for the analysis of genome-wide DNA methylation patterns (Table 1). We note that self-declared ethnicity differed considerably between the FASD and control participants, necessitating stringent statistical corrections, as described below. 
Table 1 Characteristics of the NeuroDevNet FASD cohort

\begin{tabular}{lll}
\hline & FASD cases & Controls \\
\hline$N$ & 110 & 96 \\
Age & $11.55 \pm 3.37$ & $11.28 \pm 3.38$ \\
Sex & & \\
$\quad$ Male (\%) & 41 & 47 \\
Female (\%) & 59 & 53 \\
Self-declared ethnicity & & \\
Caucasian & $27 \%(48 \%)^{\mathrm{a}}$ & $91 \%(96 \%)$ \\
Other & $73 \%(52 \%)$ & $9 \%(4 \%)$ \\
\hline
\end{tabular}

a Percentages in brackets include participants with mixed ethnicity including Caucasian

\section{Children with FASD displayed altered DNA methylation patterns}

The DNA methylation profiles of BECs from the complete NeuroDevNet cohort were assessed using the Illumina HumanMethylation450 array, which assays DNA methylation at 485,512 sites across the human genome. Following quality control and normalization to remove probes with bad detection $p$-values and low bead counts, or those associated with sex chromosomes, SNPs, and polymorphic CpGs, 404,430 sites remained in the final dataset of 206 samples [42]. Although BECs typically represent a relatively homogenous population of cells, they can occasionally be contaminated by white blood cells during collection, thus possibly affecting the results of differential DNA methylation analyses [43]. To assess whether BEC from the present study had high levels of contamination, principal component analysis of BECs and blood samples obtained from GEO (GSE42861) was performed. This analysis did not reveal any blood contamination in our dataset, as evidenced by the distant clustering of samples from both tissue types (Additional file 1: Figure S1). Having thus established that cellular heterogeneity was unlikely to confound our results, we next set out to identify alterations in DNA methylation patterns specific to the FASD group. For this, differential DNA methylation analysis using a two-group design was coupled with surrogate variable analysis (SVA), which corrects for batch effects and any other undesirable variation in the data. This analysis identified 1661 differentially methylated (DM) CpG sites between the FASD group and controls at a false discovery rate $(\mathrm{FDR})<0.05$, indicating substantial differences in DNA methylation patterns between the two groups. However, self-declared ethnicity in the cohort was strongly confounded with FASD status (Table 1). Given that ethnicity has been associated with altered DNA methylation levels, these differences could potentially drive alterations in DNA methylation at these 1661 DM CpG sites [19-21].
Ethnic background correction identified FASD-specific DNA methylation patterns

To account for ethnicity on a genetic basis, the Illumina HumanOmni2.5 array was used to obtain genotypes at nearly 2.4 million single nucleotide polymorphisms (SNPs) for each child. Participants were clustered by multi-dimensional scaling (MDS) of genotypic data along with publicly accessible data from the HapMap project [44]. Linear regression of the first four genetic clusters from this analysis with the SVs revealed little correlation with the majority of DNA methylation variation, suggesting that further correction for differences in ethnicity was required to isolate the effects of PAE beyond ethnicity (Additional file 1: Figure S2). As such, individuals clustering within the larger and more genetically homogeneous subgroup were selected for further analysis, consisting of 49 FASD cases and 87 controls (Table 2; Additional file 1: Figure S3; Additional file 3: Table S2). Differential DNA methylation analysis was performed on the more genetically homogeneous subsample to isolate the effects of PAE in the absence of an ethnic confound. In support of less ethnicity-related effects in this subsample, SVA identified fewer SVs compared to the full dataset. Furthermore, the results from DNA methylation analysis in this subgroup displayed only a moderate correlation with those obtained from the full sample (Spearman rank correlation: 0.43 ), suggesting that ethnicity indeed may have influenced differential DNA methylation patterns in the full cohort, despite our efforts to use SVA to remove the effects of ethnicity. Therefore, the subsample was used to filter out ethnically confounded CpG loci to obtain a subset of DM sites unbiased for ethnicity (Fig. 1). More specifically, the top 5242 probes (unadjusted $p$-value $<0.01$ ) in the genetically homogeneous subsample were selected as a conservative set of differentially methylated $\mathrm{CpG}$ sites between FASD cases and controls that were unaffected by ethnic background. This set was compared to the 1661 DM sites identified in the full sample, and only

Table 2 Characteristics of the more genetically homogenous subsample

\begin{tabular}{lll}
\hline & FASD cases & Controls \\
\hline$N$ & 49 & 87 \\
Age & $11.29 \pm 3.16$ & $11.29 \pm 3.37$ \\
Sex & & \\
Male (\%) & 43 & 41 \\
Female (\%) & 57 & 59 \\
Self-declared ethnicity & & \\
Caucasian & $51 \%(76 \%)^{\mathrm{a}}$ & $93 \%(97 \%)$ \\
Other & $49 \%(24 \%)$ & $7 \%(3 \%)$ \\
\hline
\end{tabular}

a Percentages in brackets include participants with mixed ethnicity including Caucasian 


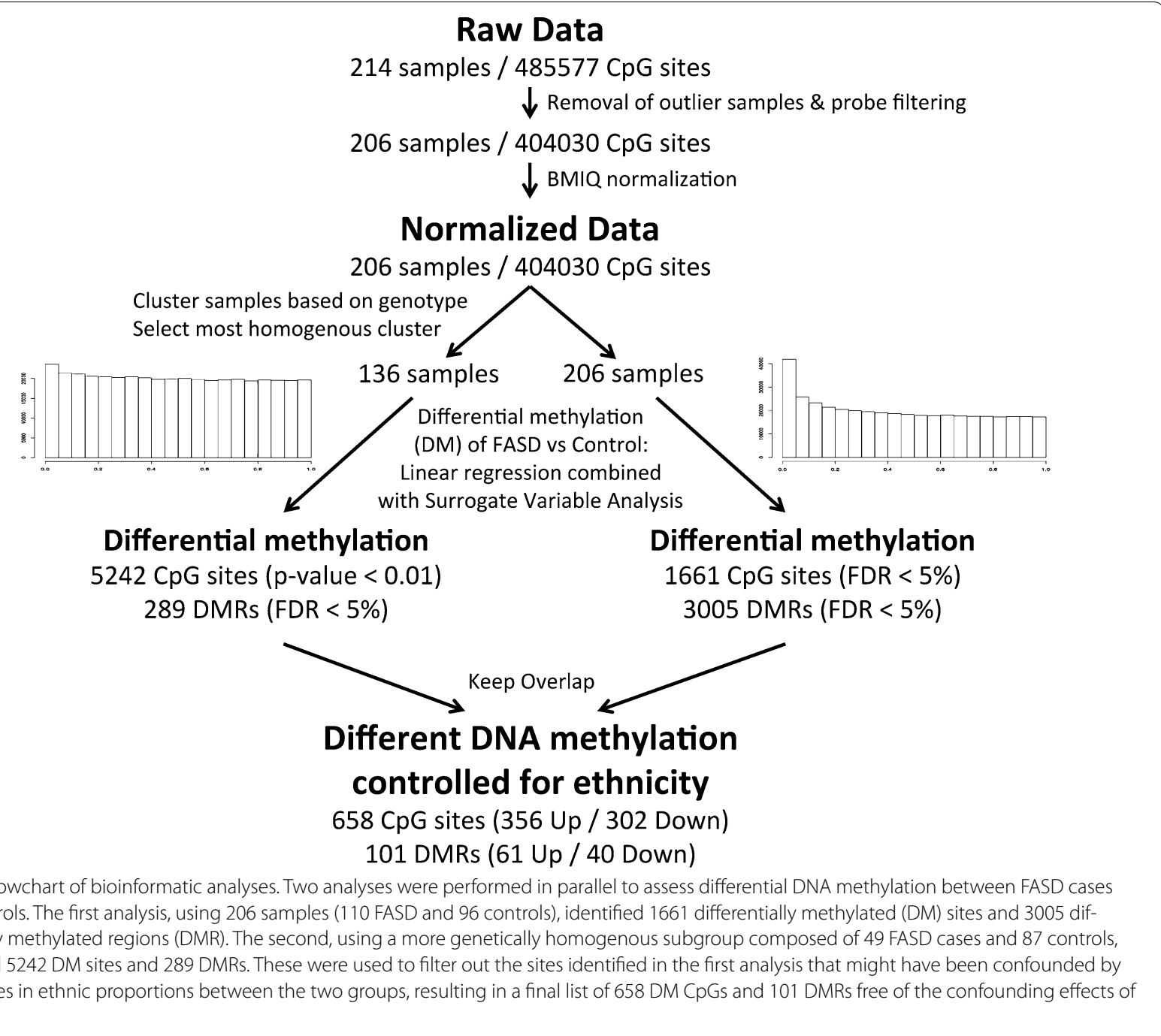

the probes present in both lists were considered specific effects of FASD, unlikely to be related to effects of ethnicity. Following this strategy, a final list of 658 DM CpG sites significantly altered in FASD cases was obtained at an FDR $<0.05$ (Additional file 2: Table S1), composed of 356 down-methylated and 302 up-methylated sites compared to controls (Fig. 2a, b). To determine whether this corrective analysis removed some or all effects of ethnicity, differential DNA methylation analysis was performed on FASD cases from the two main ethnic clusters from MDS to tease apart ethnicity and FASD-specific effects between the groups (Additional file 1: Supplemental methods). As expected, the ethnicity-corrected CpGs were less associated with ethnic differences in DNA methylation patterns than the uncorrected set of CpGs, as evidenced by the decreased area under the ROC curve (Additional file 1: Figure S4). Furthermore, reflecting the economic realities of our study populations, socioeconomic status (SES) scores were slightly confounded between groups $(p=0.00017$; Additional file 1: Figure S5), with the FASD group displaying lower overall scores than controls. However, the more ethnically homogeneous subgroup showed less skewing toward low SES in the FASD group ( $p=0.16$; Additional file 1: Figure S5), suggesting that the effects of SES might also have been partially accounted for during the correction for ethnic biases between groups. As such, the ethnicity-corrected set of 658 CpG loci associated with FASD was used in all subsequent analyses. The changes observed in the absolute methylation levels of these DM CpGs were relatively small, consistent with previous human studies of neurological and neurodevelopmental disorders, with percent methylation changes ranging from 0.16 to $13.1 \%$ after correction for surrogate variables (SVs) [45]. However, 


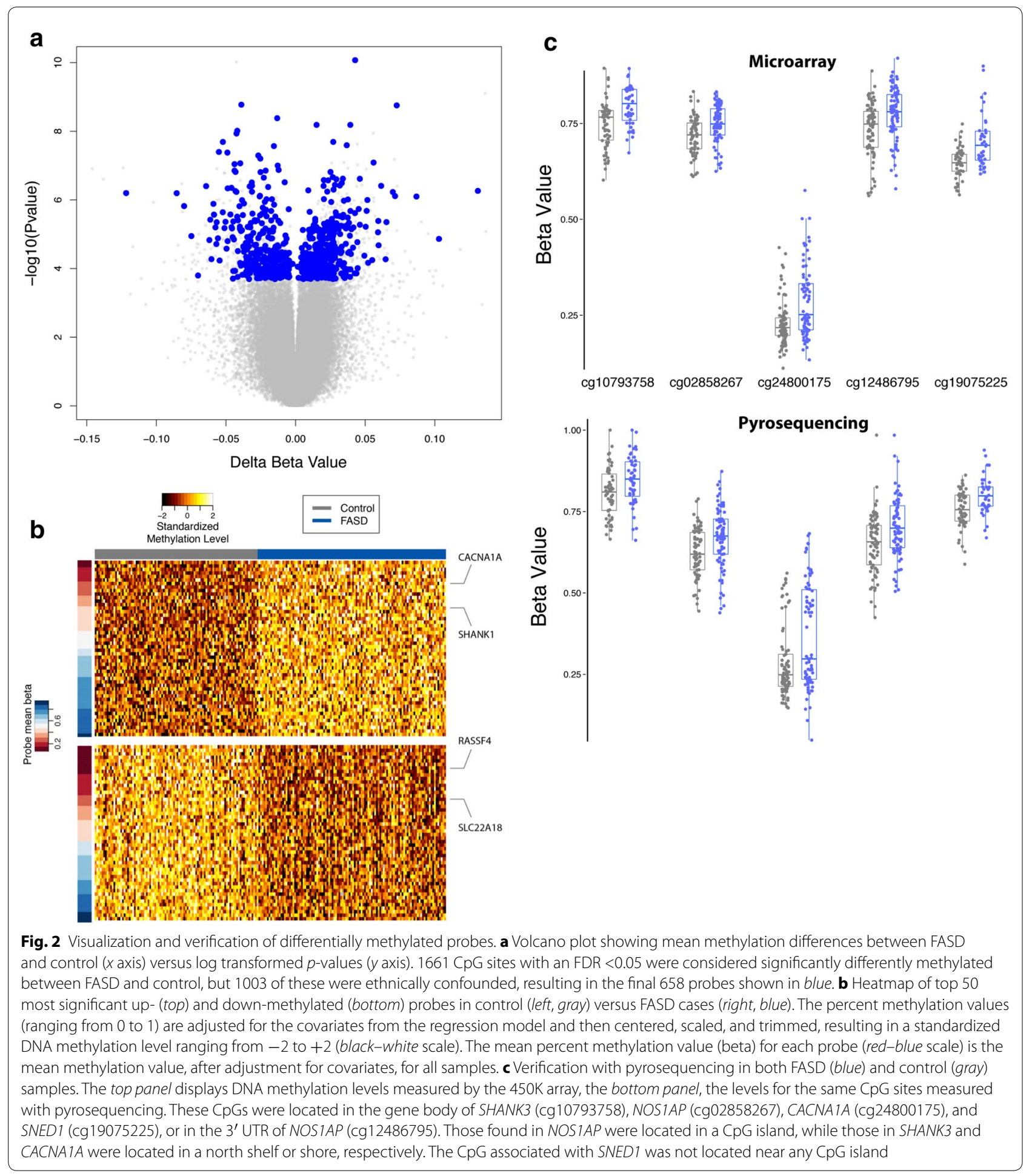

41 DM sites passed an arbitrary threshold for possible biological relevance of $>5 \%$ difference in DNA methylation levels between groups. Taken together, these results support the hypothesis that FASD is associated with altered DNA methylation patterns, largely free of identified confounding effects due to ethnicity and SES. 


\section{Technical verification of FASD DM loci by bisulfite pyrosequencing}

To ensure that the results from the differential DNA methylation analysis were not dependent on the method used to measure them, five CpG sites with a difference in percent methylation change $>5 \%$ in the vicinity of genes with potential biological relevance were selected for verification using bisulfite pyrosequencing on the same samples. Pyrosequencing results confirmed the DNA methylation levels observed on the $450 \mathrm{~K}$ array, showing similar DNA methylation levels and differences between groups for CpGs located in SHANK3, NOS1AP, CAC$N A 1 A$, and $S N E D 1$ (Fig. 2c). Pearson correlations ranged from 0.421 to 0.801 and Bland-Altman plots showed little difference when comparing both methods, suggesting a strong concordance between DNA methylation data from microarray and the different pyrosequencing methods (Additional file 1: Figure S6). Perhaps more importantly, linear regression analysis of pyrosequencing data confirmed differential DNA methylation between FASD cases and controls in this subset of biologically relevant sites, even in the absence of covariates, as the $p$-values ranged from $3.7 \mathrm{E}-04$ to $5.5 \mathrm{E}-03$. Collectively, pyrosequencing data verified the findings from the $450 \mathrm{~K}$ array, suggesting that individuals with FASD had altered DNA methylation patterns compared to typically developing children.

\section{Overlap of BEC FASD signatures with brain tissue gene expression and DNA methylation}

As alterations to DNA methylation patterns in children with FASD were identified in BECs, it is important to note that changes in peripheral tissues do not necessarily reflect alterations in a relevant tissue, such as the brain, even though these two tissues originate from the same germ layer and thus might share some epigenetic concordance [46]. Therefore, two complimentary approaches were used to obtain an approximation for the relationship of these FASD-associated DM loci to brain biology and possible the etiology of FASD. First, DM genes were compared to publically available gene expression data from 896 postmortem brain regions (Allen Institute for Brain Science) to determine whether they were expressed at biologically relevant levels in neural tissue [47]. This analysis revealed that $56 \%$ of DM genes identified in BECs displayed mRNA expression levels in the brain above the median expression for all genes, with $68 \%$ ranked in the top $2 / 3$ of the genes based on mean ranking across $\sim 900$ brain regions [48]. These findings held true whether all DM genes or only the down-/up-methylated genes were considered for analysis. Next, the FASD BEC DNA methylation patterns were compared to DNA methylation patterns from unrelated postmortem cortical brain specimens previously published by our group [48]. The overall correlation of mean DNA methylation between BEC and brain samples for all 658 DM CpGs was 0.76 (Additional file 1: Figure S7). Taken together, these results indicated that $\mathrm{BEC}$ may be a suitable surrogate tissue for brain cells and that the DM loci presented here could potentially report on biological alterations in neural tissues.

\section{FASD DM loci were enriched in regions of high DNA methylation variability}

Given that genomic location plays an important role in sculpting DNA methylation landscapes and mediating its effects, we ascertained the relative enrichment of FASD DM loci in distinct genomic features. Overall, DM probes had a significantly different distribution than the proportions present on the entire $450 \mathrm{~K}$ array (Fig. 3a; down-methylated probes: $\chi^{2}=33.63, p=2.8 \mathrm{E}-06$; upmethylated probes: $\left.\chi^{2}=13.30, p=2.1 \mathrm{E}-02\right)$. Compared to all $450 \mathrm{~K}$ probes, both down- and up-methylated CpGs in FASD cases were significantly underrepresented in CpG island cores, which generally show the least amount of variability in DNA methylation levels (down-methylated $p=1.62 \mathrm{E}-6$; up-methylated $p=7.53 \mathrm{E}-4$ ). By contrast, down-methylated sites were enriched in CpG island shores and shelves $(p=0.04 ; p=0.0003)$, which tend to be more variable than $\mathrm{CpG}$ island cores [14]. Up-methylated sites were overrepresented in non-CpG island regions $(p=0.009)$, further supporting a greater effect of PAE on malleable regions of the epigenome. Moreover, the distribution of average methylation levels for DM sites was significantly different than that of all 404,030 sites (Student's $t$ test; $p=2.5 \mathrm{E}-09$; Additional file 1: Figure S8). Further analysis of this phenomenon revealed a significant enrichment for DM CpG sites in the intermediate $20-80 \%$ range of methylation levels, while showing a concordant underrepresentation in the hypo-methylated $(<20 \%)$ and hyper-methylated $(>80 \%)$ categories (Fig. 3b) [49]. These findings suggested that DM loci in the FASD cases versus controls were mostly located in more variable regions of the epigenome.

\section{Multiple DM sites were associated with imprinted genes and the protocadherin gene cluster}

Next, the association of DM loci with different genes was assessed, with particular regards to whether some of these harbored more than one CpG differentially methylated between FASD and controls. Using genome location annotations from UCSC, the DM sites were mapped to 403 different genes. Of these, 190 were down-methylated, 208 were up-methylated, and five displayed inconsistent differences between FASD cases and controls, containing both up- and down-methylated sites, which were 


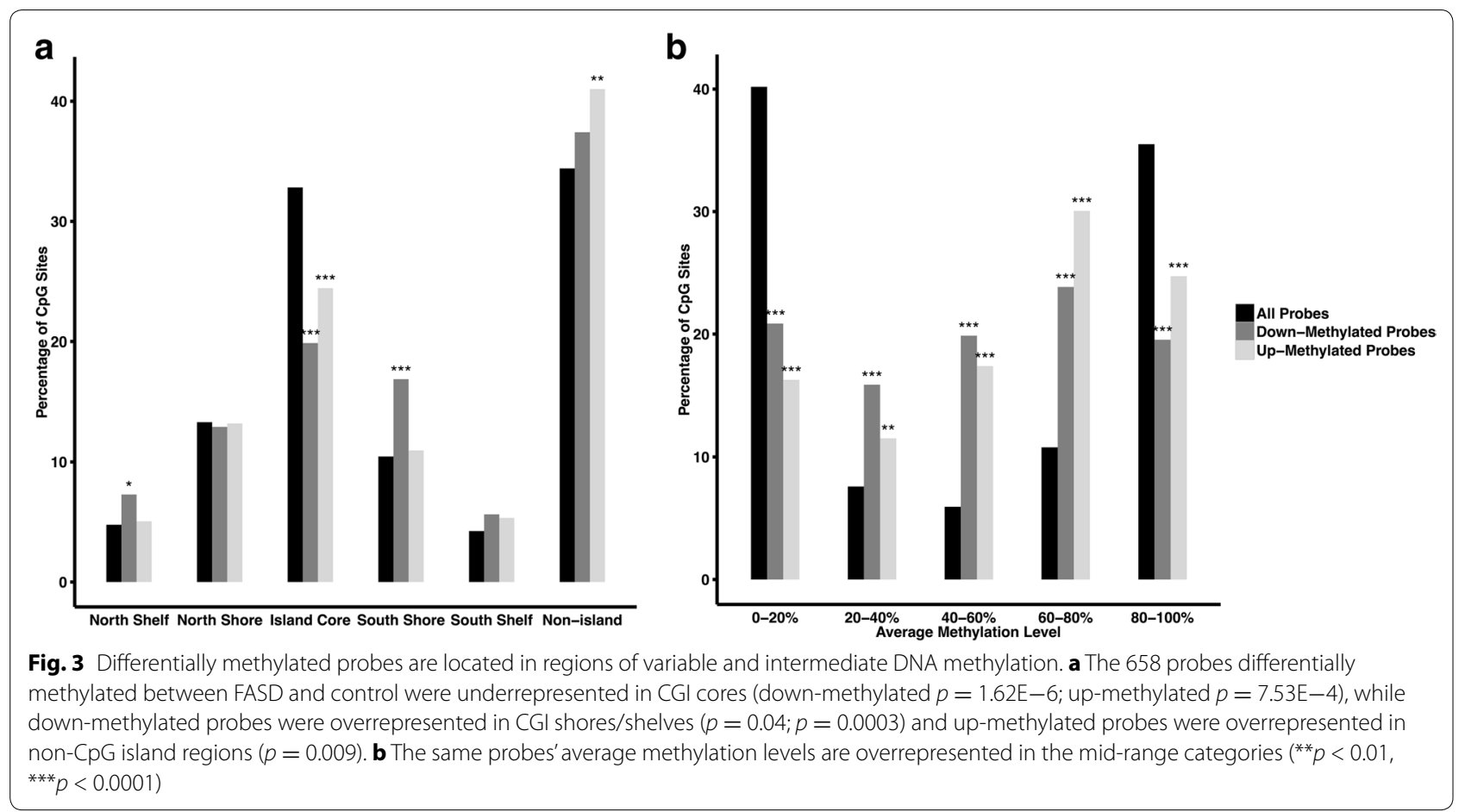

likely due to different genomic locations within the genes (Additional file 3: Table S2). The Phenocarta resource for gene-disease associations has previously curated a list of susceptibility genes for FASD, identifying 123 potential candidates from both human and animal studies of PAE [50]. However, DNA methylation analysis of the 115 FASD candidate genes assayed on the $450 \mathrm{~K}$ array did not reveal significant alterations in FASD cases. Nonetheless, twelve genes contained three or more DM loci, including several genes previously involved in studies of alcohol exposure and dependence, but not present in the Phenocarta list, such as SLC6A3 and DRD4 (Table 3) [51-54]. This short list of DM genes also showed a slight but statistically significant enrichment for imprinted genes. The geneimprint Web site (www.geneimprint. com) currently lists 96 human genes as imprinted, 80 of which were assayed on the $450 \mathrm{~K}$ array. Of these, five were differentially methylated in FASD cases versus controls (ATP1OA, CPA4, H19, KCNQ1OT1, SLC22A18), with 12 out of 15 DM CpGs showing lower methylation levels in the FASD group, which resulted in a strong enrichment for imprinted probes in the list of differentially methylated probes (Fisher's exact test; $p=1.8 \mathrm{E}-04$ ). In particular, the six CpGs located within the SLC22A18 promoter were clustered together, showing a similar pattern between FASD cases and controls, suggesting a robust regional effect of PAE on this gene's DNA methylation profile (Fig. 4). Furthermore, 15 of the $658 \mathrm{DM}$ sites were located within protocadherin genes, including six in the
PCDHB cluster, six in the PCDHGA cluster, two in the $P C D H A$ cluster, and one in $P C D H 9$. Given the presence of multiple DM CpGs within these genes, these results provide support for imprinted genes and protocadherin

Table 3 Genes containing three or more differentially methylated probes

\begin{tabular}{llll}
\hline $\begin{array}{l}\text { Gene } \\
\text { \# of probes }\end{array}$ & $\begin{array}{l}\text { Direction } \\
\text { of change }\end{array}$ & $\begin{array}{l}\text { Previous reports } \\
\text { (PMID) }\end{array}$ \\
\hline $\begin{array}{l}\text { PCDHB gene } \\
\text { cluster }\end{array}$ & 6 & Up & - \\
$\begin{array}{l}\text { PCDHGA gene } \\
\text { Cluster }\end{array}$ & 6 & Up & - \\
SLC22A18 & 6 & Down & 20009564 \\
H19 & 5 & Down & 21382472 \\
& & & 19519716 \\
& & & 19279321 \\
HLA-DPB1 & 5 & Up & 20009564 \\
DES & 4 & Down & - \\
FAM59B & 4 & Down & - \\
(GAREML) & & & - \\
SLC38A2 & 4 & Down & - \\
CAPN10 & 3 & Up & - \\
DRD4 & 3 & Down & $20009564^{\mathrm{a}}$ \\
RASSF4 & 3 & Inconsistent & - \\
SLC6A3 & 3 & Up & 18504048 \\
\hline Previous & & &
\end{tabular}

a Previous reports describe change in opposite direction 


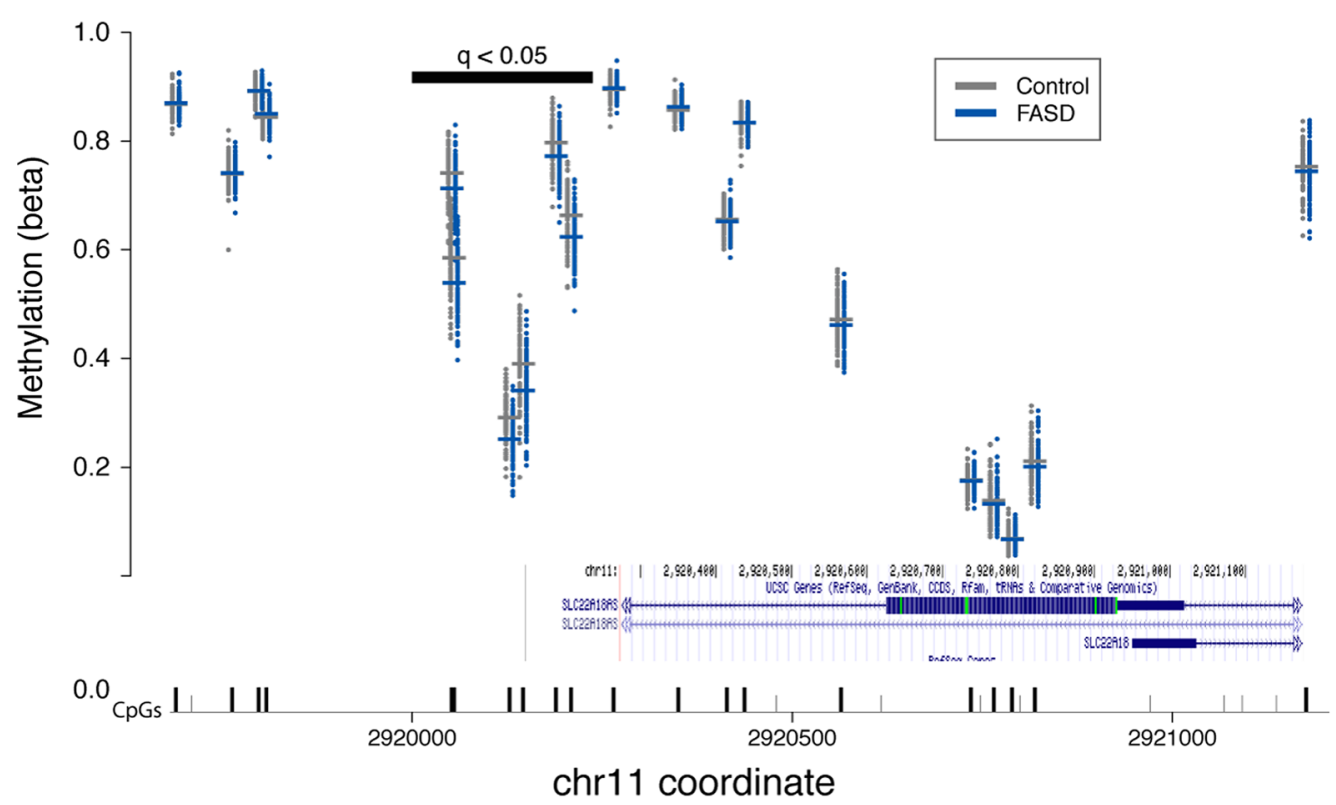

Fig. 4 Several CpGs associated with SLC22A18 displayed down-methylation in FASD cases. The covariate-adjusted DNA methylation levels for control (gray) and FASD (blue) samples are shown for SLC22A18AS (top), with the gene structure aligned (bottom). Exons are represented by blocks, and transcriptional direction is indicated by arrows. All CpG sites are noted, those present on the 450K array are black while CpGs not present are gray. The six significantly differentially methylated probes located in the SLC22A18 promoter region are indicated with the horizontal black bar [FDRadjusted $p$-value $(q)<0.05]$

clusters as strong candidates for the effects of PAE on the epigenome.

\section{Association of FASD DM loci with neurodevelopmental processes and disorders}

In order to identify broad biological processes associated with altered DNA methylation patterns in FASD children, gene function enrichment analysis was performed on the dataset. As no significant results were obtained from the entire list of DM genes following multiple-test correction, the analysis was performed separately on both the up- and down-methylated gene lists. Given that the up-methylated gene list included several members of the protocadherin beta $(P C D H B)$ and gamma A (PCDHGA) clusters, which are not differentiated by gene function annotations, a single gene from each cluster was conserved for the analysis to avoid any redundancy that may skew the results. As such, only 199 up-methylated genes and 190 down-methylated genes were analyzed for functional annotations using the overrepresentation analysis (ORA) tool in ErmineJ [55]. While no significant results were obtained using the gene ontology (GO) annotation with the list of downmethylated genes, the up-methylated gene list showed enrichment for genes associated with neurodevelopmental processes (Table 4 ), such as neuron parts (20 genes;
$\mathrm{FDR}=0.051)$ and projections (19 genes; FDR $=0.082)$ $[50,56]$. Furthermore, using the Phenocarta annotation for associations with diseases, the list of up-methylated genes was enriched for several neurodevelopmental disorders (Table 5), including "epilepsy syndrome" (15 genes; FDR $=0.081)$, "autistic disorder" (12 genes; FDR $=0.092$ ), and "anxiety disorder" (eight genes; $\mathrm{FDR}=0.071)[50,56]$. Of note, the up-methylated genes were also marginally enriched for genes associated with substance-related disorder (15 genes; FDR $=0.192$ ). To further examine the regulatory circuitry associated with FASD DM genes, a co-expression analysis of the up-methylated genes across 282 human expression microarray experiments, spanning multiple tissues and experimental conditions, was performed using the Gemma web tools [57]. Of the up-methylated genes, 86 could be included in the co-expression network (Fig. 5). The most strongly co-expressed pair was caldesmon 1 (CALD1)-Palladin (PALLD), which are both cytoskeleton-associated proteins [58]. In addition, a small cluster of the network showed co-expression of several genes (NRXN1, CACNA1A,CDH10, and others) associated with autism and/or epilepsy. Taken together, these findings suggest that altered DNA methylation patterns may potentially relate to the neurobiological deficits of children with FASD. 
Table 4 Gene ontology function enrichment in genes up-methylated in FASD

\begin{tabular}{|c|c|c|c|c|}
\hline GO name & GO ID & $p$-value & FDR & Genes \\
\hline Neuron part & GO:0097458 & $1.38 \mathrm{E}-05$ & 0.051 & $\begin{array}{l}\text { ATP2B2, CDH13, GABRB1, HEPACAM, KCNAB2, KCND3, KCTD16, NFASC, NMU, NRSN1, NRXN1, } \\
\text { P2RX7, PAM, ROBO3, SHANK1, SHANK3, SLC6A1, SLC6A3, SLC8A1, TIAM2, UCN3 }\end{array}$ \\
\hline Vocalization behavior & GO:0071625 & $1.18 \mathrm{E}-05$ & 0.066 & NRXN1, SHANK1, SHANK3 \\
\hline Neuron projection & GO:0043005 & $7.31 \mathrm{E}-06$ & 0.082 & $\begin{array}{l}\text { CDH13, GABRB1, HEPACAM, KCNAB2, KCND3, NFASC, NMU, NRSN1, NRXN1, P2RX7, PAM, } \\
\text { ROBO3, SHANK1, SHANK3, SLC6A1, SLC6A3, SLC8A1, TIAM2, UCN3 }\end{array}$ \\
\hline
\end{tabular}

Table 5 Disease association enrichment in genes up-methylated in FASD

\begin{tabular}{|c|c|c|c|c|}
\hline Disease name & Disease ID & $p$-value & FDR & Genes \\
\hline Anxiety disorder & DOID_2030 & $1.44 \mathrm{E}-04$ & 0.071 & CRHR2, CYP3A4, GRM8, NOS1AP, P2RX7, PAM, SHANK1, SLC6A3 \\
\hline Pervasive developmental disorder & DOID_0060040 & $1.15 E-04$ & 0.076 & $\begin{array}{l}\text { AGAP1, ARID1B, ATP2B2, ATP10A, CDH10, DCUN1D1, DPP6, ESRRB, GABRB1, } \\
\text { GRM8, HEPACAM, NOS1AP, NRXN1, PCDHAC2, ROBO3, SDK1, SHANK1, } \\
\text { SHANK3, SLC6A3, ST8SIA2 }\end{array}$ \\
\hline Epilepsy syndrome & DOID_1826 & 2.07E-04 & 0.081 & $\begin{array}{l}\text { BRD2, CACNA1A, CCR3, CIT, GJD2, GRM1, GRM8, KCNAB2, NRXN1, NTNG2, } \\
\text { P2RX7, PAM, SLC6A1, SLC6A3, SLC8A1 }\end{array}$ \\
\hline Autistic disorder & DOID_12849 & $4.70 \mathrm{E}-05$ & 0.092 & $\begin{array}{l}\text { AGAP1, ATP10A, CDH10, GABRB1, GRM8, HEPACAM, NOS1AP, NRXN1, ROBO3, } \\
\text { SHANK1, SHANK3, ST8SIA2 }\end{array}$ \\
\hline Autism spectrum disorder & DOID_0060041 & $1.01 \mathrm{E}-04$ & 0.099 & $\begin{array}{l}\text { AGAP1, ARID1B, ATP2B2, ATP10A, CDH10, DCUN1D1, DPP6, ESRRB, GABRB1, } \\
\text { GRM8, HEPACAM, NOS1AP, NRXN1, PCDHAC2, ROBO3, SDK1, SHANK1, } \\
\text { SHANK3, SLC6A3, ST8SIA2 }\end{array}$ \\
\hline Substance-related disorder & DOID_303 & $6.85 E-04$ & 0.192 & $\begin{array}{l}\text { ADARB2, ANPEP, CACNA1A, CDH13, CRHR2, FRMD4A, GRM8, KCND3, KISS1R, } \\
\text { NMU, NRXN1, SLC6A1, SLC6A3, TIAM2, TRPM4 }\end{array}$ \\
\hline
\end{tabular}

\section{Differentially methylated regions were identified between the FASD group and controls}

To complement the site-specific analysis of differential DNA methylation, which identified several genes with multiple DM CpGs, we next attempted to identify broader patterns of differential DNA methylation using an unbiased approach. Specifically, the identification of region-specific clusters of DM CpGs between children with FASD and controls was performed using DMRcate, an established method that uses a Gaussian kernel smoother to identify regions of differential DNA methylation [59]. In the full dataset, 3005 differentially methylated regions (DMRs) containing two or more CpGs were identified at an FDR $<0.05$, while in the more homogeneous subset of samples, 289 statistically significant DMRs were identified between groups. Using the same approach to correct for the confounding effects of ethnicity as described in the site-specific analysis, 101 DMRs unbiased by ethnicity were uncovered between individuals with FASD and controls (Additional file 4: Table S3). On average, these spanned 471 nucleotides, with lower and upper limits of 31 and $2450 \mathrm{bp}$, respectively. DMRs each contained between 2 and 20 CpGs assayed on the $450 \mathrm{~K}$ array, for a total of 504 unique sites, 75 of which were also identified in the first differential methylation analysis. Of these, 74 overlapped with
95 different genes, and 27 were located in intergenic regions. Of those associated with genes, 25 overlapped with promoter regions (within $1500 \mathrm{bp}$ of the transcriptional start site), 23 with the $5^{\prime}$ UTR, 16 with the first exon, 49 with the gene body, and six with the $3^{\prime}$ UTR, as annotated from the hg19 genome assembly. Moreover, 15 of the top DMRs associated with one or more genes overlapped with those containing multiple DM CpGs in the previous analysis, including SLC22A18, SLC38A2, HLA-DBP1, and NOS1AP (Table 6; Fig. 6a, b). These showed the same direction of change across the entire DMR, consistent with the individual CpG differential methylation analysis and verification by pyrosequencing, in the case of NOS1AP. Moreover, two DMRs were identified within the protocadherin genes, with eight CpGs spanning the PCDHGA and PCDHGB clusters and four CpGs spanning the promoter of $P C D H 12$, further supporting a potential role for the protocadherin genes in FASD. Importantly, in addition to the genes overlapping with the previous DM analysis, several additional DM genes were identified through this analysis, including UCN3 and ITGAL, key components of the stress and immune response, respectively (Fig. 6c, d). Taken together, these results suggested that the effects of PAE on the DNA methylation went beyond single CpG loci to affect broader chromosomal neighborhoods. 


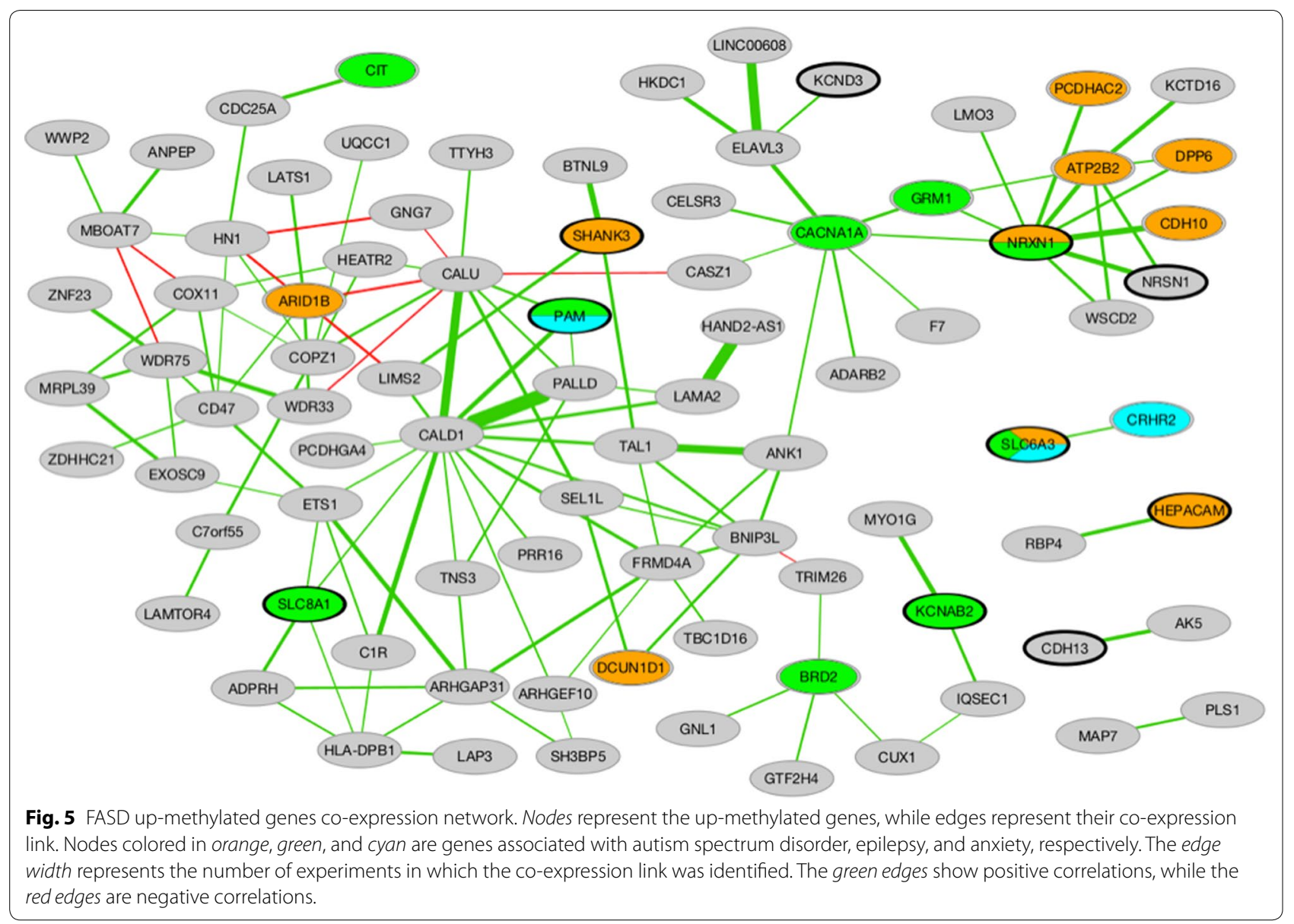

\section{Discussion}

This study aimed to assess the effects of PAE on genomewide DNA methylation patterns and identify an epigenetic signature of FASD, using a large cohort of human subjects. Significant changes to the DNA methylation profiles in BECs of children with FASD compared to age- and sex-matched typically developing controls were identified, with 658 CpGs displaying significantly altered DNA methylation levels, of which 41 had a $>5 \%$ methylation change. Moreover, 101 DMRs containing two or more sequential DM CpGs were identified throughout the genome, spanning 95 different genes, overlapping with several from the initial differential methylation analysis at single CpG level. The majority of DM genes were highly expressed in postmortem brain samples from the Allen Brain Institute. Moreover, BEC and independent cortical samples showed relatively high concordance of DNA methylation levels. As discussed in more detail below, several lines of evidence converge to support the validity of our data. First, a number of DM sites and regions were identified within genes and pathways previously associated with PAE. Second, novel DM sites and regions tended to be involved in pathways implicated in functional deficits of FASD. Third, broader patterns related to altered neurodevelopmental disorders were identified in sets and networks of genes associated with FASD in our study.

Differential DNA methylation analysis in our casecontrol study comparing children with FASD to children with typical development replicated several associations from previous studies of PAE. One of the most striking similarities is the altered DNA methylation patterns observed in imprinted genes. Several studies have demonstrated the effect of PAE on the H19 imprinted gene in both mice and humans [31, 60, 61]. A genome-wide DNA methylation study in mouse embryos exposed to ethanol also identified significant changes within several imprinted genes including both $H 19$ and SLC22A18 [38]. Results from our study further confirmed these findings, as five down-methylated probes in $\mathrm{H} 19$ and six in SLC22A18 were altered in the FASD cohort, with the latter being identified as a broader DMR as well. Given that imprinting plays a key role in the regulation of normal growth and development, its alteration by alcohol 
Table 6 Top 30 gene-annotated differentially methylated regions associated with FASD

\begin{tabular}{|c|c|c|c|c|c|c|c|c|}
\hline Gene symbol(s) & DMR location & Chr & Start position & End position & \# of probes & Min FDR & Mean FDR & Max beta $\mathrm{FC}^{\mathrm{a}}$ \\
\hline HLA-DPB1 & Body & 6 & 33047056 & 33049505 & 17 & $2.59 E-50$ & $1.61 \mathrm{E}-06$ & 0.087 \\
\hline SLC22A18, SLC22A18AS & $\begin{array}{l}\text { Body, TSS1500, TSS200, 5' } \\
\text { UTR }\end{array}$ & 11 & 2919689 & 2921176 & 20 & $1.21 \mathrm{E}-29$ & 1.46E-05 & -0.049 \\
\hline PPP1R2P1 & Body & 6 & 32846924 & 32847845 & 18 & $1.81 E-20$ & $9.39 E-10$ & 0.026 \\
\hline SLC38A2 & TSS1500 & 12 & 46767132 & 46768016 & 8 & $1.98 \mathrm{E}-16$ & $9.78 \mathrm{E}-09$ & -0.039 \\
\hline HKR1 & $\begin{array}{l}\text { TSS1500, TSS200, 1st exon, } \\
\text { 5' UTR }\end{array}$ & 19 & 37825307 & 37825679 & 7 & $7.51 \mathrm{E}-16$ & $9.51 \mathrm{E}-16$ & 0.022 \\
\hline WDR52 & $\begin{array}{l}\text { 5' UTR, 1st exon, TSS200, } \\
\text { TSS1500 }\end{array}$ & 3 & 113160071 & 113160821 & 10 & $1.34 \mathrm{E}-14$ & $6.02 E-13$ & -0.037 \\
\hline C3orf24 & $\begin{array}{l}\text { 5' UTR, 1st exon, TSS200, } \\
\text { TSS1500 }\end{array}$ & 3 & 10149466 & 10150487 & 11 & $4.41 \mathrm{E}-13$ & $1.88 \mathrm{E}-11$ & 0.034 \\
\hline NOS1AP & Body, $3^{\prime}$ UTR & 1 & 162336877 & 162337375 & 5 & $4.69 \mathrm{E}-13$ & $8.79 E-13$ & 0.039 \\
\hline KCNAB2 & $5^{\prime}$ UTR & 1 & 6093770 & 6094993 & 6 & $9.78 \mathrm{E}-13$ & $2.86 \mathrm{E}-07$ & 0.026 \\
\hline F7 & TSS1500, TSS200, Body & 13 & 113759771 & 113760286 & 6 & $1.55 \mathrm{E}-10$ & $1.96 \mathrm{E}-10$ & 0.029 \\
\hline IFT140, TMEM204 & Body & 16 & 1598866 & 1599150 & 4 & $1.81 E-10$ & $4.34 \mathrm{E}-10$ & -0.036 \\
\hline RGL3 & Body & 19 & 11517079 & 11517436 & 4 & $3.06 \mathrm{E}-10$ & $5.34 \mathrm{E}-10$ & 0.036 \\
\hline STRA6 & $\begin{array}{l}\text { 5' UTR, 1st exon, TSS200, } \\
\text { TSS1500 }\end{array}$ & 15 & 74494781 & 74496040 & 12 & $4.80 E-10$ & 1.06E-04 & 0.035 \\
\hline TXNRD1, EID3 & $\begin{array}{l}\text { 5' UTR, Body, TSS1500, } \\
\text { TSS200, 1st exon }\end{array}$ & 12 & 104697193 & 104697983 & 11 & $5.49 \mathrm{E}-10$ & $3.98 \mathrm{E}-08$ & 0.024 \\
\hline RNMTL1 & Body, $3^{\prime}$ UTR & 17 & 695156 & 695661 & 3 & $5.77 \mathrm{E}-10$ & $3.23 \mathrm{E}-09$ & -0.026 \\
\hline C22orf42 & Body, TSS200 & 22 & 32554848 & 32555310 & 5 & $7.95 E-10$ & $7.91 \mathrm{E}-09$ & 0.022 \\
\hline RADIL & Body & 7 & 4869981 & 4870162 & 3 & $2.40 E-09$ & $2.48 \mathrm{E}-09$ & 0.026 \\
\hline ITGAL & Body & 16 & 30485383 & 30485966 & 6 & 7.18E-09 & $5.13 \mathrm{E}-08$ & 0.022 \\
\hline ZNF710 & $5^{\prime} \cup T R$ & 15 & 90547692 & 90548043 & 3 & $4.18 \mathrm{E}-08$ & $5.44 \mathrm{E}-07$ & -0.023 \\
\hline $\begin{array}{l}\text { PCDHA7, PCDHAC2, } \\
\text { PCDHA12, PCDHA6, } \\
\text { PCDHA10, PCDHA4, } \\
\text { PCDHA11, PCDHA8, } \\
\text { PCDHA1, PCDHA2, } \\
\text { PCDHA9, PCDHA13, } \\
\text { PCDHA5, PCDHAC1, } \\
\text { PCDHA3 }\end{array}$ & Body, TSS1500 & 5 & 140344290 & 140344745 & 4 & 4.73E-08 & $1.20 \mathrm{E}-07$ & 0.019 \\
\hline MAL2 & TSS200, 1st exon, body & 8 & 120220410 & 120221797 & 8 & $1.26 \mathrm{E}-07$ & $2.35 E-03$ & -0.022 \\
\hline UCN3 & $\begin{array}{l}\text { TSS1500, TSS200, 1st exon, } \\
\text { 5' UTR }\end{array}$ & 10 & 5406543 & 5407020 & 8 & $1.32 \mathrm{E}-07$ & $3.03 \mathrm{E}-07$ & 0.016 \\
\hline HKDC1 & TSS1500, 5' UTR, 1st exon & 10 & 70979777 & 70980067 & 4 & $1.37 \mathrm{E}-07$ & $1.40 \mathrm{E}-07$ & 0.023 \\
\hline ARHGEF19 & Body & 1 & 16533422 & 16534579 & 8 & $1.88 \mathrm{E}-07$ & $1.11 E-04$ & -0.035 \\
\hline LOC154822 & Body & 7 & 158815555 & 158816392 & 3 & $2.36 \mathrm{E}-07$ & $1.90 \mathrm{E}-05$ & -0.043 \\
\hline NDST4 & $\begin{array}{l}\text { 1st exon, 5' UTR, TSS200, } \\
\text { TSS1500 }\end{array}$ & 4 & 116034871 & 116035232 & 4 & $5.96 \mathrm{E}-07$ & $6.45 \mathrm{E}-07$ & 0.031 \\
\hline SNED1 & Body & 2 & 242009513 & 242009588 & 2 & $6.41 \mathrm{E}-07$ & $6.48 \mathrm{E}-07$ & 0.040 \\
\hline PRKDC & Body & 8 & 48739161 & 48739256 & 2 & $7.94 \mathrm{E}-07$ & $8.04 \mathrm{E}-07$ & -0.045 \\
\hline CASZ1 & $5^{\prime}$ UTR & 1 & 10847541 & 10847594 & 2 & $2.92 \mathrm{E}-06$ & $2.92 \mathrm{E}-06$ & 0.025 \\
\hline HEATR2 & Body & 7 & 807596 & 809109 & 9 & $3.11 E-06$ & $3.69 E-04$ & 0.036 \\
\hline
\end{tabular}

${ }^{a}$ Max fold changes (FC) represented in percent methylation change (beta) in DNA methylation levels of FASD compared to control

exposure could be a factor in the neurodevelopmental defects observed in children with FASD [62]. Furthermore, the only other study of genome-wide DNA methylation patterns in individuals with FASD also identified several DM protocadherin genes within the alpha, beta, and gamma clusters, though only one $\mathrm{CpG}$ overlapped with the results presented here [40]. The differences in specific CpGs within these gene clusters between the two studies might be due to the much larger sample size of our study, as well as our use of multiple-test correction to 


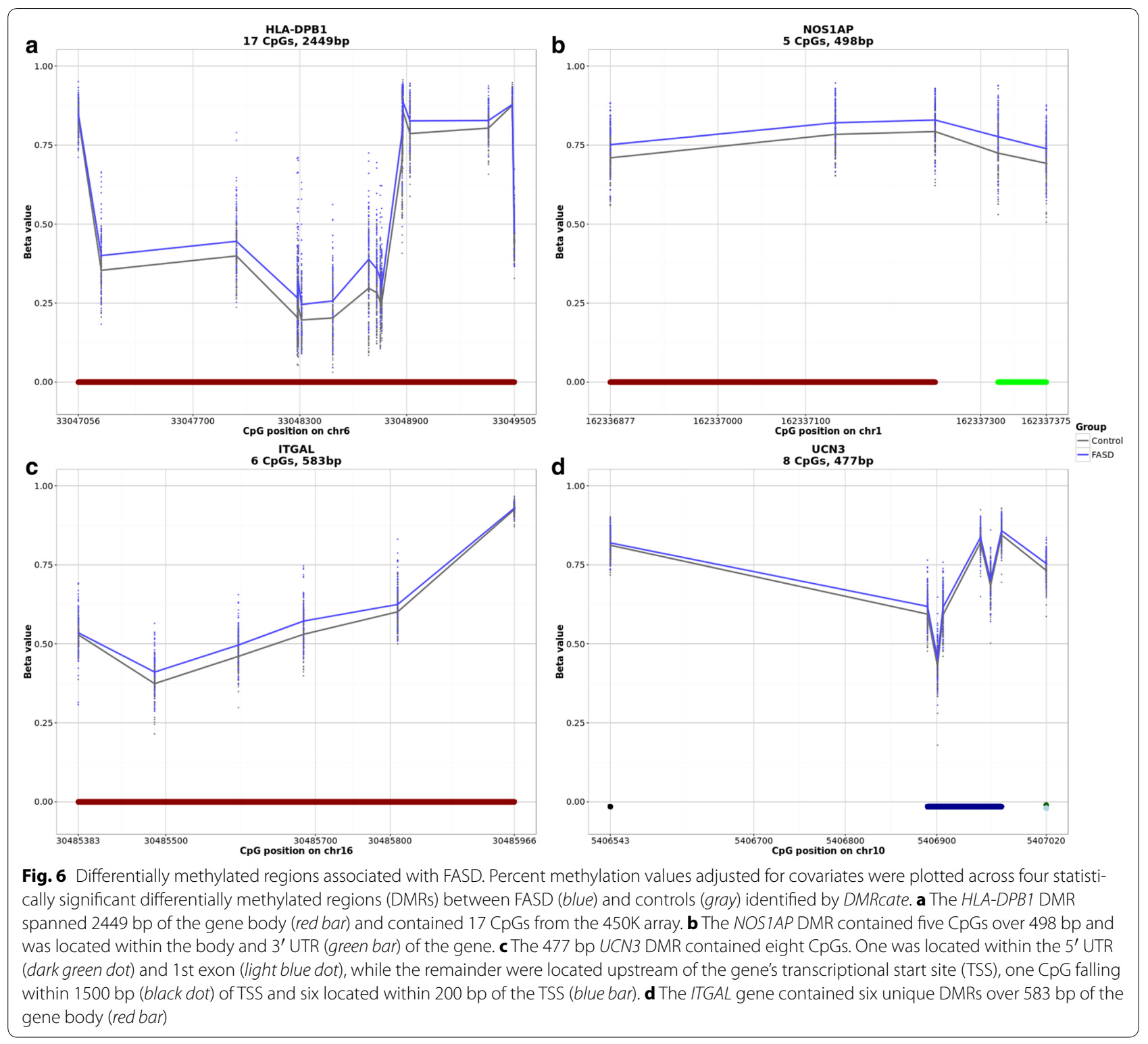

mitigate spurious patterns of differential DNA methylation associated with the FASD group. However, we note that the single CpG site from our study that overlapped with the previous findings (cg21117330) was located in PCDHGA 8 and displayed the same direction of change between FASD cases and controls and thus might represent a robust and reproducible effect of PAE.

In addition to genes previously identified in studies of PAE, DNA methylation changes were also uncovered in a number of additional genes with functional relevance to the deficits observed in FASD. More specifically, analysis of DM probes and regions identified altered DNA methylation patterns within genes related to the immune response, such as HLA-DPB1, a HLA class II histocompatibility antigen, and ITGAL (or CD11A), the integrin alpha $\mathrm{L}$ chain. Given that children with FASD often present with numerous deficits in immune function, epigenetic alterations of these genes might reflect functionally relevant underlying biology [63]. A DMR between FASD cases and controls was also identified in $U C N 3$, an antagonist of the CRF type 2 receptor, which plays a key role in the stress response. As this gene acts downstream of stress signaling pathways, this alteration might be linked to altered basal levels of corticosterone found in individuals with FASD $[1,64]$. Finally, two members of the dopaminergic system, SLC6A3 and DRD4, each contained three differentially methylated CpGs in FASD cases compared to controls. Both of these genes 
have also been proposed as modifiers and/or risk factors in alcohol abuse disorders and attention deficit disorder, and thus might potentially play a role in the deficits of attention and executive function in children with FASD $[53,54]$.

Moving beyond alterations in specific genes related to PAE, broader associations to neurodevelopmental processes and disorders were identified in genes containing differentially methylated CpGs. In particular, the gene co-expression network contained a small sub-network of genes associated with autism and/or epilepsy, and upmethylated genes in FASD cases were enriched for functions related to neurodevelopmental disorders. These results could reflect the pleiotropy of these genes, or perhaps their involvement in developmental functions dysregulated in neurodevelopmental disorders with partially overlapping phenotypes. As many of these genes were also functionally enriched for neuron parts and projections, they could influence processes necessary for typical brain development and partially underlie some deficits observed in children with FASD and other neurodevelopmental disorders.

Comparing epigenetic patterns associated with FASD and autism presented an interesting conundrum. While we identified a small sub-network of genes associated with autism and/or epilepsy in our analysis of the FASDrelated gene co-expression network, this relationship did not extend to the level of individual CpGs. Comparing the 14 DM genes from BECs recently reported to be associated with autism spectrum disorder, we did not find any overlap with the DM loci identified in our study of FASD children [46]. The differences between the gene lists may reflect the different origins and phenotypes between the conditions, or that the effects of PAE are more easily identifiable in peripheral tissue than those of autism, or simply false positives and/or false negatives. Regardless, these results imply that at the single CpG level, genes showing differences in DNA methylation between FASD cases and controls are reflective of FASD-specific alterations, rather than broad neurodevelopmental functions.

Although it is tempting to speculate that our collective results may be partially related to the functional deficits observed in FASD, it is important to consider that the DNA methylation patterns were derived from BECs. We feel that this concern is partially mitigated by our finding of the majority of DM genes in BECs being consistently expressed in the brain and by the DNA methylation patterns in neural tissue displaying high correlation with those in BEC. Moreover, it has been noted by others that BECs might be a good surrogate tissue for human DNA methylation studies, as both buccal and brain cells are derived from the ectoderm [65]. Lastly, while our study did not measure DNA methylation in additional tissues, evidence from animal models is emerging to support lasting alterations to both epigenetic and gene expression patterns in neural tissue following PAE [39, 66-68]. Nevertheless, our results must be interpreted with caution in the context of neurodevelopment, as additional studies in postmortem samples from humans are required to rigorously assess the concordance of epigenetic changes associated with FASD between peripheral and central tissues.

A further challenge in the interpretation of alterations to DNA methylation patterns in FASD cases versus controls lies in the small effect sizes of environmental exposures on the epigenome. Although the small DNA methylation changes observed here are consistent with genome-wide DNA methylation studies in other neurodevelopmental and psychiatric disorders, it is unclear whether such small changes can have a strong effect on cellular functions [45, 46, 69]. However, slight changes accumulating in several genes involved in similar processes could combine to have strong effects on biological processes. For instance, as many of the up-methylated genes were co-expressed, small alterations to multiple members of this network could potentially affect the biological functions they regulate.

While our data are very consistent with published work in human epigenome-wide association studies, it is of course possible that the relatively small changes to DNA methylation levels reflect biological biases or even technical noise [69]. These could originate from a variety of sources, which we attempted to address to the best of our abilities. For example, although differences in cell-type composition can play an important role in driving DNA methylation variation, no contamination of the BEC from the present study with white blood cells was identified, suggesting that differences in cell-type composition likely did not affect the observed alterations to DNA methylation patterns in the FASD group. In addition to differences in cell types, differing postnatal environments between groups might also influence the observed DNA methylation patterns, skewing the results to represent possibly confounding variables other than PAE, such as diet, SES, and postnatal alcohol exposure. However, the majority of children in the FASD group were living in foster or adoptive homes, rather than the biological family, which hopefully would reduce differences in the rates of alcohol use or food security between groups. By contrast, SES scores were slightly confounded between groups, although this effect was partially mitigated by the focus on the more ethnically homogeneous subgroup, which showed less skewing toward low SES in the FASD cases. Finally, we feel that potential technical issues were reduced through the use of strict quality control and statistical procedures to eliminate unwanted variation in the data. As such, the technical validity of our approach was 
supported by the verification of five DM loci by bisulfite pyrosequencing, the gold standard for targeted DNA methylation analysis.

We note that although most biological and technical issues were addressed by our study design and methods, a particular caveat in the identification of DM loci was manifested by the imbalance in ethnicity across FASD cases and control groups. Other studies have included ethnicity as a covariate during linear modeling to correct for its effects, but no significant DM probes were identified using this approach in our study, as FASD status was confounded with ethnic background (Additional file 1: Supplemental methods). Given that self-reports do not always accurately assess ethnicity, SNP genotyping data were used to objectively assign participants to different ethnic groups, based on HapMap samples of known ethnicity. This analysis resulted in the identification of a more homogeneous subgroup of samples, which was used as a comparative control to filter out the influences of ethnicity and related effects, such as SES and cultural confounders, on differential DNA methylation within FASD cases. In turn, this strategy facilitated the removal of ethnically biased probes from the original DM loci, resulting in the successful identification DM CpG sites specific to children with FASD and not confounded for ethnicity. Given the prevalence of ethnically diverse populations in large-scale studies of DNA methylation, this unique approach driven by genetic stratification of subgroups might prove a useful way of dealing with the effects of ethnicity in case-control studies beyond the one presented here.

\section{Summary and conclusions}

Despite the recognition of FAS over 40 years ago, PAE remains a leading cause of developmental disability in the developed world. While several animal studies have investigated the role of epigenetic mechanisms in the context of PAE, most human studies have been limited to alcohol consumption and dependence in adults, or a small cohort of children with FASD [40, 52, 70, 71]. As such, this study is the single largest investigation of genome-wide DNA methylation patterns in children with FASD. While one of the greatest challenges with this large cohort was the ethnicity imbalance between the FASD and control groups, ethnic background correction reduced this confound and allowed the reliable identification of 658 DM CpG sites specific to children with FASD. Although the effect size of changes was small in some cases, 41 sites displayed a $>5 \%$ change in DNA methylation, which is consistent with previous studies and may reflect the subtle effects of PAE on the epigenome. We also identified 101 DMRs containing two or more DM CpGs, located within 95 different genes and spanning promoter regions, gene bodies, and both $3^{\prime}$ and $5^{\prime}$ UTRs. While these data were collected from BEC, rather than neural tissue, the vast majority of DM genes were highly expressed in the brain, suggesting a potential concordance between peripheral and central tissues. These alterations occurred in several genes previously implicated with PAE and altered neurodevelopment, and displayed functional enrichments for neural process and neurodevelopmental disorders. Although it will be essential to validate these changes in separate cohorts from a different population, these findings provide initial insight into the molecular mechanisms underlying the effects of PAE on children and present a potential role for role for DNA methylation in the etiology of FASD.

\section{Methods}

\section{Participants and samples}

Ethics for this project were reviewed and approved by the "Children's and Women's Research Ethics BoardClinical" (H10-01149). Children with FASD and ageand sex-matched typically developing children were recruited from multiple FASD diagnostic clinics across Canada, where saliva samples and BECs were collected for genotyping and DNA methylation analysis, respectively [41]. All experimental procedures were reviewed and approved by the Health Research Ethics Boards at Queen's University, University of Alberta, Children's Hospital of Eastern Ontario, University of Manitoba, and the University of British Columbia. Written informed consent was obtained from a parent or legal guardian, and assent was obtained from each child before study participation. The majority of clinics used previously described guidelines for the diagnosis of FASD [72]. Briefly, samples were collected from 112 FASD and 102 age- and sex-matched control children aged between 5 and 18 (Table 1). Saliva samples were collected using the Oragene DNA kit (DNA Genotek Inc., Ontario, Canada) according to the manufacturer's instructions. BECs were collected using the Isohelix buccal swabs and DriCapsule (Cell Projects Ltd., Kent, UK). To collect buccal cells, the swab was inserted into the participants' mouth and rubbed firmly against the inside of the left cheek for $1 \mathrm{~min}$. The swab was then placed into a sterile tube with a Dri-Capsule and the tube sealed. An identical procedure was followed for the right cheek. Participants did not have any dental work performed $48 \mathrm{~h}$ prior to collection, and no food was consumed $<60 \mathrm{~min}$ prior to collection to avoid contamination.

\section{DNA methylation 450K assay}

DNA was extracted from buccal swabs using the Isohelix DNA isolation kit (Cell Projects, Kent, UK). Seven hundred and fifty nanograms of genomic DNA was subjected 
to bisulfite conversion using the Zymo EZ DNA Methylation Kit (Zymo Research, Irvine, CA, USA), which converts DNA methylation information into sequence base differences by deaminating unmethylated cytosines to uracil while leaving methylated cytosines unchanged. One hundred and sixty nanograms of converted DNA was applied to the HumanMethylation450 BeadChip array from Illumina (450K array), which enables the simultaneous quantitative measurements of 485,512 CpG sites across the human genome, following the manufacturer's instructions. Chips were scanned on an Illumina HiScan, with the 214 samples run in two batches and each containing an equal number of FASD and control samples, randomly distributed across the chips. Two pairs of technical replicates were included and showed a Pearson correlation coefficient $r>0.996$ in both cases, highlighting the technology's reproducibility.

\section{DNA methylation data quality control and normalization}

The raw DNA methylation data were subjected to a set of rigorous quality controls, first of the samples and then of the probes. Of the 214 initial samples, eight were removed from the final dataset due to various quality and concordance issues. Of these, five were removed based on poor quality data, which was identified through skewed internal controls and/or $\geq 5 \%$ of probes with a detection $p$-value $>0.05$. One sample was removed due to a gross chromosomal abnormality identified in the genotyping and DNA methylation data. The genotypes of the samples, based on the 65 SNP probes contained on the $450 \mathrm{~K}$ array, were compared to the genotypes from the SNP arrays. The genotypes were highly correlated for all samples (Pearson correlation coefficient $r>0.9$ ), except one, which was excluded from further analyses. Finally, as a pair of monozygotic twins was present in the control group, only one of their samples was chosen at random and retained in the analysis to remove any genetic bias. Next, probes were removed from the dataset according to the following criteria: (1) probes on $\mathrm{X}$ and $\mathrm{Y}$ chromosomes $(N=11,648)$; (2) SNP probes $(N=65)$; (3) probes with beadcount $<3$ in $5 \%$ of samples $(N=3029)$; (4) probes with $1 \%$ of samples with a detection $p$-value $>0.05$ $(N=10,163)$; or $(5)$ probes with a polymorphic CpG and nonspecific probes as defined by the Price annotation $(N=20,869$ SNP-CpG and 41,937 nonspecific probes; [42]). A final filtering step was performed to set the methylation values to NA for any remaining probe-sample pair where beadcount $<3$ or detection $p$-value $>0.05$. Data normalization was performed using the beta-mixture quantile normalization method on the final dataset, composed of 206 samples (110 FASD and 96 controls) and 404,030 probes [73]. All analyses were performed using $M$ values, which represent the $\log 2$ ratio of methylated/ unmethylated, where negative values indicate $<50 \%$ methylation and positive values indicate more than $50 \%$ methylation [74]. Percent methylation changes (betavalues) were used in graphical representations of the data and indicate the percentage of methylation calculated by methylated/(methylated + unmethylated), ranging from 0 (fully unmethylated) to 1 (fully methylated).

\section{Differential methylation analysis}

Given that DNA methylation changes are typically small and that unknown sources of variation, including cellular heterogeneity, may influence the data, SVA was performed to identify SVs representative of unwanted heterogeneity using the SVA package in R [75]. Using DNA methylation data from all 206 samples, SVA identified 15 SVs not associated with clinical status (FASD vs control), which, as expected, were only partially correlated with known covariates (Additional file 1: Supplemental methods, Figure S2). Linear regression analysis was performed on the dataset with the limma package in $\mathrm{R}$, utilizing a model that included clinical status and all identified SVs as covariates [76]. Statistically significant differences between groups were required to show a false discovery rate $(\mathrm{FDR})<0.05$ following multiple-test correction by the Benjamini-Hochberg method [77]. Further evaluation of potential biological significance was assessed by mean percent DNA methylation differences between FASD and controls.

\section{Analysis of effects due to familial and diagnosis status}

As the cohort included several sets of siblings and cousins, a sensitivity analysis was performed to identify potential family effects in the dataset. However, little effect of familial origin was observed, indicating that the presence of families in the cohort did not significantly impact the study's results or require statistical correction (Additional file 1: Supplemental methods). Furthermore, this cohort also included children with PAE that were not formally diagnosed with FASD (27 children). As such, additional differential DNA methylation analyses were performed on the two individual subgroups of FASD cases compared to controls (Additional file 1: Supplemental methods). However, as these did not reveal any significant differences between diagnosed FASD cases and PAE children, the PAE cases were included in the FASD group for all analyses.

\section{Genotyping}

Genomic DNA was extracted from saliva samples following standard procedures. Briefly, 161 DNA samples were genotyped for 2,443,177 markers using the Infinium HumanOmni2.5-Quad v1.0 BeadChip (Illumina Inc., San Diego, CA, USA) and 54 samples were genotyped for 
2,379,855 markers using the Infinium HumanOmni2.5-8 v1.0 BeadChip (Illumina Inc., San Diego, CA, USA) according to the manufacturer's protocol. For both microarrays, $200 \mathrm{ng}$ of DNA ( $4 \mu \mathrm{L}$ at $50 \mathrm{ng} / \mu \mathrm{L})$ was independently amplified, labeled, and hybridized to BeadChips and then scanned with default settings using the Illumina iScan. Analysis and intra-chip normalization of resulting image files were performed using Illumina's GenomeStudio Genotyping Module software v.2011 with default parameters. Genotype calls were generated using the Illumina-provided genotype cluster definitions files (HumanOmni2.5-4v1_H.egt and HumanOmni2.5-8v1_C. egt generated using HapMap project DNA samples) with a Gencall cutoff of 0.15 . Only the 2,368,900 common SNPs were used for analysis. pyGenClean v1.2.2 and PLINK v1.07 were used for quality control and genetic data cleanup process, respectively. SNPs with completion rate $<98 \%$, uninformative $(\mathrm{MAF}=0)$ and failed for Hardy-Weinberg equilibrium exact test ( $p$-value $<2.9 \times 10^{-8}$ ) were removed. Samples with completion rate $<95 \%$ were excluded.

\section{Subsample definition}

MDS was performed on the participants' genotype data including 83 founder individuals from the Caucasian population (CEU), 186 from the Japanese and Han Chinese population (JPT-CHB), and 88 from the Yoruba population (YRI) (HapMap; [78]).

All 195 samples that had both genotyping and DNA methylation data were hierarchically clustered based on the first four principal components from the MDS analysis. One individual of African descent was excluded because of their unique ethnicity compared to the rest. All other samples clustered in two groups: Cluster $1=136$ samples (49 FASD:87 controls) and Cluster $2=58$ samples (53 FASD:5 control) (Additional file 1: Figure S3). Cluster 1 was selected as the more balanced subsample, in terms of both ethnicity and cases versus controls, for further analysis (see Fig. 1 for a summary of the bioinformatic analyses).

\section{Ethnic group adjustment}

Differential DNA methylation analysis was performed as previously described on the more genetically homogenous subsample defined as "Cluster 1" in the MDS analysis above to identify difference between FASD cases and controls. SVA using this subsample identified 11 SVs that were added as covariates in linear modeling, as described for the full sample. Ethnically confounded probes were explored in more detail to ensure that the adjustment was performing as expected (Additional file 1: Supplemental methods, Figure S4). In addition, the inclusion of principal components from the MDS analysis into the regression model to correct for ethnicity was also explored. However, as ethnicity was confounded with the phenotype of interest, direct correction in the model also removed the signal of interest (Additional file 1: Supplemental methods).

\section{DNA methylation pyrosequencing assay}

Bisulfite pyrosequencing assays were designed with PyroMark Assay Design 2.0 (Qiagen; Additional file 5: Table S4). The regions of interest were amplified by PCR using the HotstarTaq DNA polymerase kit (Qiagen) as follows: 15 min at $95^{\circ} \mathrm{C}, 45$ cycles of $95^{\circ} \mathrm{C}$ for $30 \mathrm{~s}, 58^{\circ} \mathrm{C}$ for $30 \mathrm{~s}$, and $72{ }^{\circ} \mathrm{C}$ for $30 \mathrm{~s}$, and a $5 \mathrm{~min} 72^{\circ} \mathrm{C}$ final extension step. For pyrosequencing, single-stranded DNA was prepared from the PCR product with the Pyromark ${ }^{\mathrm{TM}}$ Vacuum Prep Workstation (Qiagen), and the sequencing was performed using sequencing primers on a Pyromark ${ }^{\mathrm{TM}} \mathrm{Q} 96$ MD pyrosequencer (Qiagen). The quantitative levels of methylation for each $\mathrm{CpG}$ dinucleotide were calculated with Pyro Q-CpG software (Qiagen).

\section{Brain concordance analysis}

Human brain blood DNA methylation data from a previously published cohort were used to assess concordance, which was calculated as the Spearman correlation coefficient of DNA methylation at all CpGs between healthy human blood and brain [48]. Human brain microarray data were obtained from the Allen Brain Atlas (http:// human.brain-map.org/static/download), which contains normalized expression values for 58,692 probes and 896 brain regions from six individuals. Probes were ranked based on their average expression level for each brain region separately, and the mean was calculated across all brain regions. All 29,191 genes assayed (which included 389 out of our 404 differentially methylated genes) were sorted based on their highest ranked probe.

\section{CpG island distribution}

The probes categorization into "North Shelf," "North Shore," "Core Island," "South Shore," "South Shelf," or "Non-island" was based on the Illumina "RELATION TO_UCSC_CPG_ISLAND" annotation. The expected counts were calculated with the 404,030 probes remaining after filtering. Statistics were calculated using multinomial goodness-of-fit Chi-square test. As a post hoc test to evaluate which category is driving the effect, additional Chi-square tests were run on each category versus the sum of all of the other categories.

\section{Functional enrichment analysis}

The list of imprinted genes was extracted from http://www.geneimprint.com/site/genes-by-species. Homo+sapiens.imprinted-All (Additional file 6: Table 
S5), which includes 80 genes with at least one probe among the 404,030 probes remaining after filtering (3035 probes total). The Illumina "UCSC_REFGENE_NAME" annotation was used to map the probes to genes (479 out of 658 DM probes had such annotation and could be mapped). In the event of probes mapping to several genes, the gene with the closest transcription start site (TSS) was selected using the Price annotation [42]. The ORA tool of ermineJ (version 3.0.2) was used to identify gene function enrichment in the list of up- and downmethylated genes including the GO annotations molecular function, biological process, and cellular component [55]. The ermineJ ORA tool was set with the following parameters: $\max$ gene set size $=1000$; $\min$ gene set size $=2$; background genes $=$ all genes mapping to the 404,030 probes remaining after filtering.

\section{Co-expression analysis}

The Gemma tools and database for meta-analysis of functional genomics data were used to perform a coexpression analysis based on existing studies [57]. The methods used by Gemma have been previously described [79]. Datasets were obtained from public sources, primarily the Gene Expression Omnibus [80]. For each dataset included in the meta-analysis, the Pearson correlation matrix of gene co-expression profiles was computed. Thresholds were applied for statistical significance of correlation, and the resulting sparse co-expression networks were aggregated across datasets. The degree to which a link is replicated across studies is a measure of its reliability; a threshold was set based on a benchmark permutation-based analysis, scaled to the number of datasets aggregated. Using the Gemma online tools, a coexpression network was extracted for the 199 up-methylated genes in the master set of microarray experiments for human (282 usable experiments across multiple tissues and experimental conditions) at the stringency recommended by the software, and visualized the results in Cytoscape [81]. The resulting network shows the coexpression relationship of the genes in the input list only.

\section{Differentially methylated region analysis}

The identification of DMRs was performed using previously established guidelines and the DMRcate package in $\mathrm{R}[59,82]$. Briefly, results from linear modeling with SVs were analyzed using a Gaussian kernel smoother with a bandwidth of $1000 \mathrm{bp}$ and scaling factor of 2 to model all CpG sites in the genome in parallel and identify broad regions of differential DNA methylation. $p$-values were corrected for multiple testing using the $\mathrm{BH}$ method, and an FDR cutoff of 0.05 was used to select significant probes between the FASD and control groups. DMRs were then assigned by clustering significant $\mathrm{CpGs}$ located within $1000 \mathrm{bp}$ windows that contained two or more CpGs. This analysis was performed on both the full dataset and the more ethnically homogeneous subset of individuals, and the final list of DMRs was obtained through the same process as previously described in the differential methylation analysis. Genomic locations for all DMRs were assigned using the Illumina hg19 annotation.

\section{Additional files}

Additional file 1. Supplementary methods include additional information on SVA, familial effects analysis, PAE vs FASD analyses, and further investigation of ethnicity-related effects on DNA methylation. Fig S1 displays the principal component analysis of BEC versus blood samples. Fig S2 shows the correlation of SVs with different variables. Fig S3 shows the clustering of samples based on genotype. Fig $\$ 4$ shows the effect of ethnicity adjustment on significantly DM probes. Fig S5 shows the distribution of SES scores between FASD and control groups. Fig S6 shows the correlation between pyrosequencing and 450K array results. Fig S7 shows the correlation of BEC and brain DNA methylation. Fig 58 shows the distribution of DNA methylation levels. Fig 59 shows the distribution of $p$-values for PAE, FASD, and control contrasts (described in additional file 1).

Additional file 2: Table S1. Table of differentially methylated probes. Additional file 3: Table S2. Table of genes with multiple differentially methylated probes.

Additional file 4: Table S3. Table of differentially methylated regions. Additional file 5: Table S4. Table of pyrosequencing primers.

Additional file 6: Table S5. Table of imprinted genes in humans.

\section{Abbreviations}

450K array: Illumina HumanMethylation450 BeadChip array; BEC: buccal epithelial cell; CpG: cytosine-guanine dinucleotide; CGI: CpG island; DM: differentially methylated; DMR: differentially methylated region; FASD: fetal alcohol spectrum disorder; FDR: false discovery rate; GO: gene ontology; MDS: multi-dimensional scaling; mQTL: methylation quantitative trait loci; ORA: overrepresentation analysis; PAE: prenatal alcohol exposure; ROC: receiver operating characteristic; SNP: single nucleotide polymorphism; SEP: socioeconomic position; SV: surrogate variable; SVA: surrogate variable analysis; TSS: transcription start site; UCSC: University of California, Santa Cruz Genome Browser; UTR: untranslated region.

\section{Authors' contributions}

EPC performed the data processing and differential methylation analysis and wrote part of the manuscript. AAL performed the DMR analysis, assisted with data interpretation and significance, and wrote part of the manuscript. JLM and SMM ran the 450K arrays. RE performed the brain concordance analysis. $A B, S P, L P L P$, and MPD ran the SNP arrays and processed those data. MJJ, MSC, AEC, JNR, PP, and MSK provided expert advice in the study design, data interpretation, and manuscript writing. All authors read and approved the final manuscript.

\footnotetext{
Author details

${ }^{1}$ Centre for High-Throughput Biology, University of British Columbia, Vancouver, BC, Canada. ${ }^{2}$ Department of Medical Genetics, Centre for Molecular Medicine and Therapeutics, Child and Family Research Institute, University of British Columbia, Vancouver, BC, Canada. ${ }^{3}$ Beaulieu-Saucier Pharmacogenomics Centre, Montreal Heart Institute, Université de Montréal, Montreal, QC, Canada. ${ }^{4}$ Brain Research Centre, University of British Columbia, Vancouver, BC, Canada. ${ }^{5}$ Department of Pediatrics and Child Health, Faculty of Medicine, University of Manitoba, Winnipeg, MB, Canada. ${ }^{6}$ Department of Biochemistry and Medical Genetics, Faculty of Medicine, University of Manitoba, Winnipeg, MB, Canada. ${ }^{7}$ Faculty of Medicine, Université de Montréal, Montreal, QC,
} 
Canada. ${ }^{8}$ Centre for Neuroscience Studies, Queen's University, Kingston, ON, Canada. ${ }^{9}$ Human Early Learning Partnership, School of Population and Public Health, University of British Columbia, Vancouver, British Columbia, Canada.

\section{Acknowledgements}

We would like to thank the following cohort leads for each collection site: Dr. Sarah Nikkel (Ottawa, ON, Canada), Dr. Dawa Samdup (Kingston, ON, Canada), Dr. Ana Hanlon-Dearman (Winnipeg, MB, Canada), Ms. Audrey McFarlane (Cold Lake, AB, Canada), Dr. Gail Andrew (Edmonton, AB, Canada), Dr. Christine Loock (Vancouver, BC, Canada), and Dr. Tim Oberlander (Vancouver, BC, Canada). This work was supported by NeuroDevNet, which is funded by the Networks of Centres of Excellence, a program of the federal government to advance science and technology (to MSK and PP). MJJ was supported by a Mining for Miracles Fellowship from the Child and Family Research Institute. MSK is the Canada Research Chair in Social Epigenetics. AAL was partially supported by NeuroDevNet funding awarded to Dr. Joanne Weinberg (UBC).

\section{Competing interests}

The authors declare that they have no competing interests.

Received: 19 February 2016 Accepted: 17 June 2016

Published online: 29 June 2016

\section{References}

1. Mattson SN, Crocker N, Nguyen TT. Fetal alcohol spectrum disorders: neuropsychological and behavioral features. Neuropsychol Rev. 2011;21(2):81-101.

2. Zhang $X$, Sliwowska JH, Weinberg J. Prenatal alcohol exposure and fetal programming: effects on neuroendocrine and immune function. Exp Biol Med. 2005;230:376-88.

3. Pei J, Denys K, Hughes J, Rasmussen C. Mental health issues in fetal alcohol spectrum disorder. J Ment Health. 2011;20(5):438-48.

4. Popova S, Lange S, Shield K, Mihic A, Chudley AE, Mukherjee RAS, Bekmuradov D, Rehm J. Comorbidity of fetal alcohol spectrum disorder: a systematic review and meta-analysis. Lancet. 2016:6736:1-10.

5. Pollard I. Neuropharmacology of drugs and alcohol in mother and fetus. Semin Fetal Neonatal Med. 2007;12:106-13.

6. Jones KL, Smith DW. Recognition of the fetal alcohol syndrome in early infancy. Lancet. 1973;302:999-1001.

7. Astley SJ, Clarren SK. Diagnosing the full spectrum of fetal alcoholexposed individuals: introducing the 4-digit diagnostic code. Alcohol Alcohol. 2000;35:400-10.

8. Jacobson SW, Jacobson JL, Stanton ME, Meintjes EM, Molteno CD. Biobehavioral markers of adverse effect in fetal alcohol spectrum disorders. Neuropsychol Rev. 2011;21(2):148-66.

9. Feil R, Fraga MF. Epigenetics and the environment: emerging patterns and implications. Nat Rev Genet. 2012;13:97-109.

10. Bird A. Perceptions of epigenetics. Nature. 2007;447:396-8.

11. Jones PA, Takai D. The role of DNA methylation in mammalian epigenetics. Science. 2001;293:1068-70.

12. Illingworth RS, Bird AP. CpG islands - 'a rough guide'. FEBS Lett. 2009;583:1713-20.

13. Doi A, Park IH, Wen B, Murakami P, Aryee MJ, Irizarry R, Herb B, LaddAcosta C, Rho J, Loewer S, Miller J, Schlaeger T, Daley GQ, Feinberg AP. Differential methylation of tissue- and cancer-specific CpG island shores distinguishes human induced pluripotent stem cells, embryonic stem cells and fibroblasts. Nat Genet. 2009:41:1350-3.

14. Irizarry RA, Ladd-Acosta C, Wen B, Wu Z, Montano C, Onyango P, Cui H, Gabo K, Rongione M, Webster M, Ji H, Potash JB, Sabunciyan S, Feinberg AP. The human colon cancer methylome shows similar hypo- and hypermethylation at conserved tissue-specific CpG island shores. Nat Genet. 2009;41:178-86

15. Bibikova M, Barnes B, Tsan C, Ho V, Klotzle B, Le JM, Delano D, Zhang L, Schroth GP, Gunderson KL, Fan JB, Shen R. High density DNA methylation array with single CpG site resolution. Genomics. 2011;98:288-95.

16. Jones PA. Functions of DNA methylation: islands, start sites, gene bodies and beyond. Nat Rev Genet. 2012;13(7):484-92.
17. Ziller MJ, Gu H, Müller F, Donaghey J, Tsai LT-Y, Kohlbacher O, De Jager PL, Rosen ED, Bennett DA, Bernstein BE, Gnirke A, Meissner A. Charting a dynamic DNA methylation landscape of the human genome. Nature. 2013:500:477-81.

18. Smith ZD, Meissner A. DNA methylation: roles in mammalian development. Nat Rev Genet. 2013;14:204-20.

19. Fraser HB, Lam LL, Neumann SM, Kobor MS. Population-specificity of human DNA methylation. Genome Biol. 2012;13:R8.

20. Moen EL, Zhang X, Mu W, Delaney SM, Wing C, McQuade J, Myers J, Godley LA, Dolan ME, Zhang W. Genome-wide variation of cytosine modifications between European and African populations and the implications for complex traits. Genetics. 2013;194:987-96.

21. Heyn H, Moran S, Hernando-Herraez I, Sayols S, Gomez A, Sandoval J, Monk D, Hata K, Marques-Bonet T, Wang L, Esteller M. DNA methylation contributes to natural human variation. Genome Res. 2013;23:1363-72.

22. Gutierrez-Arcelus M, Lappalainen T, Montgomery SB, Buil A, Ongen H, Yurovsky A, Bryois J, Giger T, Romano L, Planchon A, Falconnet E, Bielser D, Gagnebin M, Padioleau I, Borel C, Letourneau A, Makrythanasis P, Guipponi M, Gehrig C, Antonarakis SE, Dermitzakis ET. Passive and active DNA methylation and the interplay with genetic variation in gene regulation. Elife. 2013;2:e00523.

23. Wagner JR, Busche S, Ge B, Kwan T, Pastinen T, Blanchette M. The relationship between DNA methylation, genetic and expression inter-individual variation in untransformed human fibroblasts. Genome Biol. 2014;15:R37.

24. Banovich NE, Lan X, McVicker G, van de Geijn B, Degner JF, Blischak JD, Roux J, Pritchard JK, Gilad Y. Methylation QTLs are associated with coordinated changes in transcription factor binding, histone modifications, and gene expression levels. PLoS Genet. 2014;10:e1004663.

25. Langevin SM, Houseman EA, Christensen BC, Wiencke JK, Nelson HH, Karagas MR, Marsit CJ, Kelsey KT. The influence of aging, environmental exposures and local sequence features on the variation of DNA methylation in blood. Epigenetics. 2011;6:908-19.

26. Hanson M, Godfrey KM, Lillycrop KA, Burdge GC, Gluckman PD. Developmental plasticity and developmental origins of non-communicable disease: theoretical considerations and epigenetic mechanisms. Prog Biophys Mol Biol. 2011;106:272-80.

27. Yuen RK, Neumann SM, Fok AK, Peñaherrera MS, McFadden DE, Robinson WP, Kobor MS. Extensive epigenetic reprogramming in human somatic tissues between fetus and adult. Epigenetics Chromatin. 2011:4:7.

28. Joubert BR, Håberg SE, Nilsen RM, Wang X, Vollset SE, Murphy SK, Huang Z, Hoyo C, Midttun $\varnothing$, Cupul-Uicab LA, Ueland PM, Wu MC, Nystad W, Bell DA, Peddada SD, London SJ. 450K epigenome-wide scan identifies differential DNA methylation in newborns related to maternal smoking during pregnancy. Environ Health Perspect. 2012;120:1425-31.

29. Heijmans BT, Tobi EW, Stein AD, Putter H, Blauw GJ, Susser ES, Slagboom PE, Lumey LH. Persistent epigenetic differences associated with prenatal exposure to famine in humans. Proc Natl Acad Sci USA. 2008; 105:17046-9.

30. Haycock PC. Fetal alcohol spectrum disorders: the epigenetic perspective. Biol Reprod. 2009;81:607-17.

31. Haycock PC, Ramsay M. Exposure of mouse embryos to ethanol during preimplantation development: effect on DNA methylation in the h19 imprinting control region. Biol Reprod. 2009;81:618-27.

32. Kobor MS, Weinberg J. Focus on: epigenetics and fetal alcohol spectrum disorders. Alcohol Res Health. 2011;34:29-37.

33. Ungerer M, Knezovich J, Ramsay M. In utero alcohol exposure, epigenetic changes, and their consequences. Alcohol Res. 2013;35:37-46.

34. Laufer BI, Diehl EJ, Singh SM. Neurodevelopmental epigenetic etiologies: insights from studies on mouse models of fetal alcohol spectrum disorders. Epigenomics. 2013;5:465-8.

35. Resendiz M, Chen Y, Ozturk NC, Zhou FC. Epigenetic medicine and fetal alcohol spectrum disorders. Author Manuscr. 2013;5:73-86.

36. Ramsay M. Genetic and epigenetic insights into fetal alcohol spectrum disorders. Genome Med. 2010;2:27.

37. Garro AJ, McBeth DL, Lima V, Lieber CS. Ethanol consumption inhibits fetal DNA methylation in mice: implications for the fetal alcohol syndrome. Alcohol Clin Exp Res. 1991;15:395-8.

38. Liu Y, Balaraman Y, Wang G, Nephew KP, Zhou FC. Alcohol exposure alters DNA methylation profiles in mouse embryos at early neurulation. Epigenetics. 2009;4:500-11. 
39. Laufer BI, Mantha K, Kleiber ML, Diehl EJ, Addison SMF, Singh SM. Longlasting alterations to DNA methylation and ncRNAs could underlie the effects of fetal alcohol exposure in mice. Dis Model Mech. 2013;6:977-92.

40. Laufer BI, Kapalanga J, Castellani CA, Diehl EJ, Yan L, Singh SM. Associative DNA methylation changes in children with prenatal alcohol exposure. Epigenomics. 2015;7(8):1259-74.

41. Reynolds JN, Weinberg J, Clarren S, Beaulieu C, Rasmussen C, Kobor M, Dube M-P, Goldowitz D. Fetal alcohol spectrum disorders: gene-environment interactions, predictive biomarkers, and the relationship between structural alterations in the brain and functional outcomes. Semin Pediatr Neurol. 2011:18:49-55.

42. Price ME, Cotton AM, Lam LL, Farré P, Emberly E, Brown CJ, Robinson WP, Kobor MS. Additional annotation enhances potential for biologically-relevant analysis of the Illumina Infinium HumanMethylation450 BeadChip array. Epigenetics Chromatin. 2013;6:4.

43. Jones MJ, Farré P, McEwen LM, Macisaac JL, Watt K, Neumann SM, Emberly E, Cynader MS, Virji-Babul N, Kobor MS. Distinct DNA methylation patterns of cognitive impairment and trisomy 21 in Down syndrome. BMC Med Genomics. 2013;6:58.

44. Thorisson GA, Smith AV, Krishnan L, Stein LD. The international HapMap project web site. Genome Res. 2005;15:1592-3.

45. Ladd-Acosta C, Hansen KD, Briem E, Fallin MD, Kaufmann WE, Feinberg AP. Common DNA methylation alterations in multiple brain regions in autism. Mol Psychiatry. 2014;19(8):862-71.

46. Berko ER, Suzuki M, Beren F, Lemetre C, Alaimo CM, Calder RB, BallabanGil K, Gounder B, Kampf K, Kirschen J, Maqbool SB, Momin Z, Reynolds DM, Russo N, Shulman L, Stasiek E, Tozour J, Valicenti-McDermott M, Wang S, Abrahams BS, Hargitai J, Inbar D, Zhang Z, Buxbaum JD, Molholm S, Foxe JJ, Marion RW, Auton A, Greally JM. Mosaic epigenetic dysregulation of ectodermal cells in autism spectrum disorder. PLoS Genet. 2014;10:e1004402.

47. Hawrylycz MJ, Lein ES, Guillozet-Bongaarts AL, Shen EH, Ng L, Miller JA, van de Lagemaat LN, Smith KA, Ebbert A, Riley ZL, Abajian C, Beckmann CF, Bernard A, Bertagnolli D, Boe AF, Cartagena PM, Chakravarty MM Chapin M, Chong J, Dalley RA, Daly BD, Dang C, Datta S, Dee N, Dolbeare TA, Faber V, Feng D, Fowler DR, Goldy J, Gregor BW, et al. An anatomically comprehensive atlas of the adult human brain transcriptome. Nature. 2012:489:391-9.

48. Farré P, Jones MJ, Meaney MJ, Emberly E, Turecki G, Kobor MS. Concordant and discordant DNA methylation signatures of aging in human blood and brain. Epigenetics Chromatin. 2015;8:19.

49. Eckhardt F, Lewin J, Cortese R, Rakyan VK, Attwood J, Burger M, Burton J, Cox TV, Davies R, Down TA, Haefliger C, Horton R, Howe K, Jackson DK, Kunde J, Koenig C, Liddle J, Niblett D, Otto T, Pettett R, Seemann S, Thompson C, West T, Rogers J, Olek A, Berlin K, Beck S. DNA methylation profiling of human chromosomes 6, 20 and 22. Nat Genet. 2006;38:1378-85

50. Portales-Casamar E, Ch'ng C, Lui F, St-Georges N, Zoubarev A, Lai AY, Lee M, Kwok C, Kwok W, Tseng L, Pavlidis P. Neurocarta: aggregating and sharing disease-gene relations for the neurosciences. BMC Genomics. 2013;14:129.

51. Hillemacher T, Frieling H, Hartl T, Wilhelm J, Kornhuber J, Bleich S. Promoter specific methylation of the dopamine transporter gene is altered in alcohol dependence and associated with craving. J Psychiatr Res. 2009:43:388-92

52. Zhang H, Herman Al, Kranzler HR, Anton RF, Zhao H, Zheng W, Gelernter J. Array-based profiling of DNA methylation changes associated with alcohol dependence. Alcohol Clin Exp Res. 2013;37(Suppl. 1):E108-15.

53. Bau CH, Almeida S, Costa FT, Garcia CE, Elias EP, Ponso AC, Spode A, Hutz MH. DRD4 and DAT1 as modifying genes in alcoholism: interaction with novelty seeking on level of alcohol consumption. Mol Psychiatry. 2001:6:7-9.

54. Sánchez-Mora C, Ribasés $M$, Casas $M$, Bayés $M$, Bosch $R$, Fernàndez-Castillo N, Brunso L, Jacobsen KK, Landaas ET, Lundervold AJ, Gross-Lesch S, Kreiker S, Jacob CP, Lesch K-P, Buitelaar JK, Hoogman M, Kiemeney LALM, Kooij JJS, Mick E, Asherson P, Faraone SV, Franke B, Reif A, Johansson S, Haavik J, Ramos-Quiroga JA, Cormand B. Exploring DRD4 and its interaction with SLC6A3 as possible risk factors for adult ADHD: a meta-analysis in four European populations. Am J Med Genet Part B Neuropsychiatr Genet. 2011;156B:600-12.
55. Lee HK, Braynen W, Keshav K, Pavlidis P. ErmineJ: tool for functional analysis of gene expression data sets. BMC Bioinform. 2005;6:269.

56. Ashburner M, Ball CA, Blake JA, Botstein D, Butler H, Cherry JM, Davis AP, Dolinski K, Dwight SS, Eppig JT, Harris MA, Hill DP, Issel-Tarver L, Kasarskis A, Lewis S, Matese JC, Richardson JE, Ringwald M, Rubin GM, Sherlock G. Gene ontology: tool for the unification of biology. The gene ontology consortium. Nat Genet. 2000;25:25-9.

57. Zoubarev A, Hamer KM, Keshav KD, McCarthy EL, Santos JRC, Van Rossum T, McDonald C, Hall A, Wan X, Lim R, Gillis J, Pavlidis P. Gemma: a resource for the reuse, sharing and meta-analysis of expression profiling data. Bioinformatics. 2012;28:2272-3.

58. Jin L, Yoshida T, Ho R, Owens GK, Somlyo AV. The actin-associated protein Palladin is required for development of normal contractile properties of smooth muscle cells derived from embryoid bodies. J Biol Chem. 2009;284:2121-30.

59. Peters TJ, Buckley MJ, Statham AL, Pidsley R, Samaras K, Lord RV, Clark SJ, Molloy PL. De novo identification of differentially methylated regions in the human genome. Epigenetics Chromatin. 2015;8:6.

60. Stouder C, Somm E, Paoloni-Giacobino A. Prenatal exposure to ethanol: a specific effect on the H19 gene in sperm. Reprod Toxicol. 2011;31:507-12.

61. Ouko LA, Shantikumar K, Knezovich J, Haycock P, Schnugh DJ, Ramsay M. Effect of alcohol consumption on $\mathrm{CpG}$ methylation in the differentially methylated regions of $\mathrm{H} 19$ and IG-DMR in male gametes: implications for fetal alcohol spectrum disorders. Alcohol Clin Exp Res. 2009:33:1615-27.

62. Falls JG, Pulford DJ, Wylie AA, Jirtle RL. Genomic imprinting: implications for human disease. Am J Pathol. 1999;154:635-47.

63. Bodnar T, Weinberg J. Prenatal alcohol exposure: impact on neuroendocrine-neuroimmune networks. In: Cui C, Grandison L, Noronha A, editors. Neural-immune interactions in brain function and alcohol related disorders. New York: Springer Science+Business Media; 2013. p. 307-357.

64. Ergang P, Vodička M, Soták M, Klusoňová P, Behuliak M, Reháková L, Zach P, Pácha J. Differential impact of stress on hypothalamic-pituitary-adrenal axis: gene expression changes in Lewis and Fisher rats. Psychoneuroendocrinology. 2015;53:49-59.

65. Lowe R, Gemma C, Beyan H, Hawa MI, Bazeos A, Leslie RD, Montpetit A, Rakyan VK, Ramagopalan SV. Buccals are likely to be a more informative surrogate tissue than blood for epigenome-wide association studies. Epigenetics. 2013;8(4):445-54.

66. Lussier AA, Stepien KA, Neumann SM, Pavlidis P, Kobor MS, Weinberg J. Prenatal alcohol exposure alters steady-state and activated gene expression in the adult rat brain. Alcohol Clin Exp Res. 2015;39:251-61.

67. Kleiber ML, Laufer BI, Wright E, Diehl EJ, Singh SM. Long-term alterations to the brain transcriptome in a maternal voluntary consumption model of fetal alcohol spectrum disorders. Brain Res. 2012;1458:18-33.

68. Kleiber ML, Mantha K, Stringer RL, Singh SM. Neurodevelopmental alcohol exposure elicits long-term changes to gene expression that alter distinct molecular pathways dependent on timing of exposure. $J$ Neurodev Disord. 2013;5:6.

69. Rakyan VK, Down TA, Balding DJ, Beck S. Epigenome-wide association studies for common human diseases. Nat Rev Genet. 2011;12:529-41.

70. Zhang R, Miao Q, Wang C, Zhao R, Li W, Haile CN, Hao W, Zhang XY. Genome-wide DNA methylation analysis in alcohol dependence. Addict Biol. 2013:18:392-403.

71. Philibert RA, Plume JM, Gibbons FX, Brody GH, Beach SRH. The impact of recent alcohol use on genome wide DNA methylation signatures. Front Genet. 2012;3:54.

72. Chudley AE, Conry J, Cook JL, Loock C, Rosales T, LeBlanc N. Fetal alcohol spectrum disorder: Canadian guidelines for diagnosis. Can Med Assoc J. 2005;172(5 Suppl.):S1-21.

73. Teschendorff AE, Marabita F, Lechner M, Bartlett T, Tegner J, GomezCabrero D, Beck S. A beta-mixture quantile normalization method for correcting probe design bias in Illumina Infinium 450 k DNA methylation data. Bioinformatics. 2013;29(2):189-96.

74. Du P, Zhang X, Huang C-C, Jafari N, Kibbe WA, Hou L, Lin SM. Comparison of Beta-value and M-value methods for quantifying methylation levels by microarray analysis. BMC Bioinform. 2010;1 1:587.

75. Leek JT, Johnson WE, Parker HS, Jaffe AE, Storey JD. The sva package for removing batch effects and other unwanted variation in high-throughput experiments. Bioinformatics. 2012;28:882-3. 
76. Smyth GK. Linear models and empirical bayes methods for assessing differential expression in microarray experiments. Stat Appl Genet Mol Biol. 2004;3:3.

77. Benjamini Y, Hochberg Y. Controlling the false discovery rate: a practical and powerful approach to multiple testing. J R Stat Soc Ser B. 1995;57:289-300.

78. International HapMap 3 Consortium, Altshuler DM, Gibbs RA, Peltonen L, Altshuler DM, Gibbs RA, Peltonen L, Dermitzakis E, Schaffner SF, Yu F, Peltonen L, Dermitzakis E, Bonnen PE, Altshuler DM, Gibbs RA, de Bakker PIW, Deloukas P, Gabriel SB, Gwilliam R, Hunt S, Inouye M, Jia X, Palotie A, Parkin M, Whittaker P, Yu F, Chang K, Hawes A, Lewis LR, Ren Y, et al. Integrating common and rare genetic variation in diverse human populations. Nature. 2010;467:52-8.
79. Lee HK, Hsu AK, Sajdak J, Qin J, Pavlidis P. Coexpression analysis of human genes across many microarray data sets. Genome Res. 2004;14:1085-94.

80. Wheeler DL, Church DM, Edgar R, Federhen S, Helmberg W, Madden TL, Pontius JU, Schuler GD, Schriml LM, Sequeira E, Suzek TO, Tatusova TA, Wagner L. Database resources of the National Center for Biotechnology Information: update. Nucleic Acids Res. 2004;32(Database issue):D35-40.

81. Smoot ME, Ono K, Ruscheinski J, Wang P-L, Ideker T. Cytoscape 2.8: new features for data integration and network visualization. Bioinformatics. 2011;27:431-2

82. Peters TJ, Buckley MJ, Statham AL, Pidsley R, Samaras K, Lord RV, Clark SJ, Molloy PL. De novo identification of differentially methylated regions in the human genome. Epigenetics Chromatin. 2015;8:6.

\section{Submit your next manuscript to BioMed Central and we will help you at every step:}

- We accept pre-submission inquiries

- Our selector tool helps you to find the most relevant journal

- We provide round the clock customer support

- Convenient online submission

- Thorough peer review

- Inclusion in PubMed and all major indexing services

- Maximum visibility for your research

Submit your manuscript at www biomedcentral com/submit 